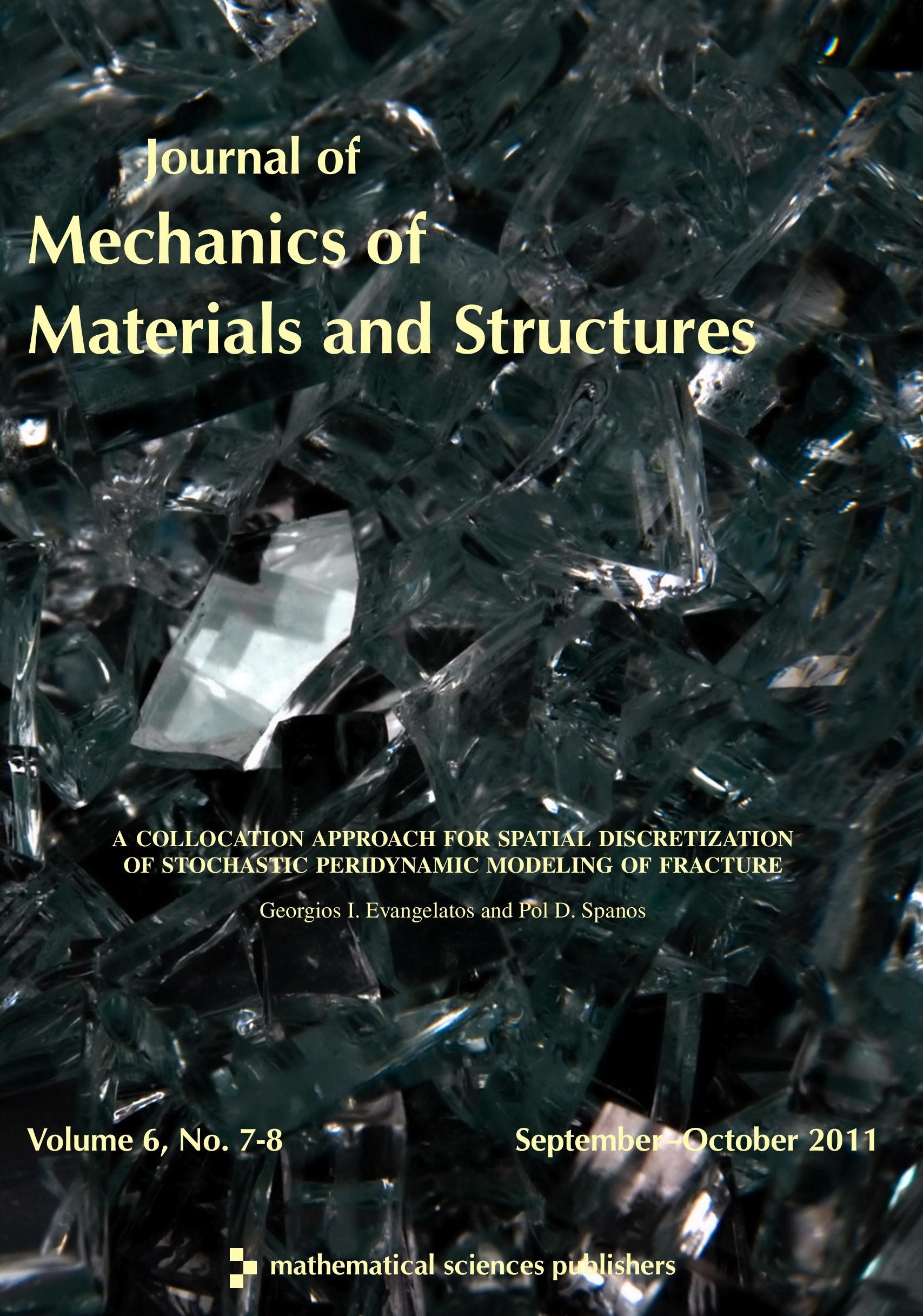




\title{
A COLLOCATION APPROACH FOR SPATIAL DISCRETIZATION OF STOCHASTIC PERIDYNAMIC MODELING OF FRACTURE
}

\author{
Georgios I. Evangelatos AND Pol D. Spanos
}

\begin{abstract}
In this paper a collocation approach is presented for spatial discretization of the partial integrodifferential equation arising in a peridynamic formulation in stochastic fracture mechanics. In the formulation nodes are distributed inside the domain forming a grid, and the inverse multiquadric radial basis functions are used as interpolation functions inside the domain. Due to this discretization the peridynamic stiffness is generated in a manner similar to the finite element method. Further, any discontinuity in the domain is included in this discretized form and affects only the peridynamic stiffness of the adjacent nodes. Using this approach as a tool, the probability density function of the energy release rate can be determined at a given crack tip point for all possible crack paths. Thus, the crack propagation direction can be probabilistically identified. This is accomplished by numerical evaluation of the requisite Neumann expansion using pertinent Monte Carlo simulations. Specific examples of applications are included.
\end{abstract}

\section{Introduction}

The problem of modeling dynamic or static systems that contain discontinuities is fundamental in mechanics. Fractured surfaces and propagating cracks are discontinuities which disrupt the domains of differential equations and create mathematical singularities. There are several approaches to dealing with these kinds of discontinuities. Two classical ones are the finite element method (FEM), with remeshing of the continuous domain, and the extended finite element method (XFEM), which circumvents the constant remeshing of the domain and uses the same mesh by simply adjusting the stiffness of the cracked element [Zi and Belytschko 2003]. Further, mesh-free techniques have been applied to the same problem with quite reasonable results [Belytschko et al. 1994; 1995]. However, all of these approaches have been based on local mechanics theory. In local mechanics theory, a single point in the medium is in direct contact with only its immediate neighboring points, and thus spatial derivatives exist in such a continuum. In nonlocal mechanics, however, a single point in the medium is in direct contact with points further away in addition to its immediate neighboring points. Nonlocal theories were initially developed in [Kröner 1967; Eringen et al. 1977] and since then several researchers have contributed to this concept.

In a pioneering publication, S. A. Silling extended the concept of nonlocal mechanics. Until then the concept of nonlocality was used as a generalization of local mechanics and it was assumed that spatial derivatives of a certain finite neighborhood around the point of interest could be used. Silling [2000] proposed a fully nonlocal peridynamic modeling in which no spatial derivatives are needed, and therefore the inherent problem of a discontinuity in the domain can be circumvented. The model involves the formulation of a partial integrodifferential equation that holds for discontinuous domains with no additional treatment such as enrichment.

Keywords: peridynamics, fracture mechanics, radial basis functions, stochastic medium, collocation method. 
Since this pioneering work, several publications have focused on the application of this approach to a wide spectrum of problems. In [Silling et al. 2003] the deformation of an infinite bar was investigated using peridynamic theory. In [Silling et al. 2007] a generalization of the original peridynamics framework was proposed to extend the kinds of materials that can be modeled by peridynamic theory. In [Bobaru et al. 2009] adaptive refinement was proposed and the uniform convergence of peridynamic theory to classical mechanics was shown for dynamic and static 1D solutions when the horizon is approaching zero. In [Warren et al. 2009] the previous peridynamic theory was extended to handle Poisson ratios other than $\frac{1}{4}$ and to allow bonds to exhibit noncentral forces. In [Macek and Silling 2007] peridynamic theory was extended beyond EMU meshless formulations to FEM by incorporating truss elements. In [Bobaru 2007] the peridynamic method was used to analyze the effect of van der Waals forces on the mechanical behavior, strength, and toughness of 3D nanofiber networks. In the benchmark study, the fracture was introduced at the microstructural level using the concept of bonds; it was concluded that two main mechanisms control the deformation: fiber reorientation and fiber accretion. In [Silling and Askari 2005] a numerical mesh-free method for solving the partial integrodifferential equation arising from peridynamic theory was proposed and examples of modeling crack growth in brittle materials were presented. In [Zhou and Du 2010] a mathematically based approach for linear peridynamic FEMs was introduced.

In this paper a peridynamic modeling of systems with stochastic material properties exposed to stochastic excitations is considered. Proceeding to this task, a novel spatial discretization of the peridynamic equation is applied which allows for the stochastic extension of the model. The governing partial integrodifferential equation is treated by the Kansa collocation method [Kansa 1990a; 1990b] using inverse multiquadric (IMQ) radial basis functions (RBF). In this formulation the direction and length of the crack propagation is not governed by the nodal density and positioning of the nodes as it is in the usual mesh-free peridynamic approach. The approach is quite similar to the XFEM [Zi and Belytschko 2003] where the crack is influencing only one finite element and the stiffness of the current element through which the crack is going needs only to be determined. Further, the boundary conditions are imposed in a simpler manner than in peridynamic theory; it is quite similar to FEM. Finally, after having established a reliable collocation method to treat the deterministic problem, the stochastic problem is considered and is solved utilizing the concept proposed in the stochastic finite element method (SFEM) [Ghanem and Spanos 1991]. The reliability of the system is evaluated by calculating the probability density function (PDF) of the energy release rate around the crack tip.

\section{Peridynamic formulation}

2.1. The peridynamic partial integrodifferential equation. Consider a mechanical component having one dimension significantly smaller than the other two, and operating under an excitation in the plane defined by its two significant dimensions. Obviously, the significant displacements of the vibrating medium are lying on the same 2D plane. Next, consider nonlocal peridynamic theory for modeling the behavior of the medium. The nonlocal theory of peridynamics involves a partial integrodifferential equation for dynamic problems [Emmrich and Weckner 2007b]. Specifically, this equation is a secondorder differential equation with respect to time, and an integral equation with respect to space. In this context, the governing equation of motion of any particle inside the vibrating $2 \mathrm{D}$ medium is given by the 
equation

$$
\rho\left[\begin{array}{l}
\ddot{u}(\underline{x}, t) \\
\ddot{v}(\underline{x}, t)
\end{array}\right]+\int_{H(\underline{x})} f\left(\left[\begin{array}{l}
u(\underline{x}, t)-u(\underline{\hat{x}}, t) \\
v(\underline{x}, t)-v(\underline{\hat{x}}, t)
\end{array}\right], \underline{x}-\underline{\hat{x}}\right) d V_{\underline{\hat{x}}}=\left[\begin{array}{l}
b_{x}(\underline{x}, t) \\
b_{y}(\underline{x}, t)
\end{array}\right],
$$

where $\ddot{u}$ and $\ddot{v}$ denote the second-order derivatives of $u$ and $v$ with respect to time, and the vectors $\underline{x}$ and $\underline{\hat{x}}$ are defined by the equations

$$
\underline{x}=\left(\begin{array}{l}
x \\
y
\end{array}\right)
$$

and

$$
\underline{\hat{x}}=\left(\begin{array}{l}
\hat{x} \\
\hat{y}
\end{array}\right) \text {. }
$$

Note that $\rho$ is the material density, $b_{x}(\underline{x}, t)$ and $b_{y}(\underline{x}, t)$ are the force densities, $u(\underline{x})$ and $v(\underline{x})$ denote the displacements of the point $\underline{x}$ on the $x$ and $y$ axes respectively, $f$ denotes the force function measured in force per unit volume squared exerted on the point $\underline{x}$ by the point $\underline{\hat{x}}$, and $H(\underline{x})$ is the domain of integration. Note that the force function $f$ may depend on the partial derivatives of the displacement with respect to the directions $x$ and $y$, and thus (1) is a partial integrodifferential equation. In peridynamic theory the domain of $\underline{\hat{x}}$ is restricted by the position of $\underline{x}$ by defining the relative position

$$
\underline{\xi}=\underline{x}-\underline{\hat{x}}
$$

such that

$$
|\underline{\xi}|<\delta \text {. }
$$

The distance $\delta$ is called the horizon and represents the distance of the nonlocal approximation. The domain $H(\underline{x})$ for every given point $\underline{x}$ is defined by the equation

$$
H(\underline{x})=\{\underline{\hat{x}}:|\underline{x}-\underline{\hat{x}}|<\delta\},
$$

and yields a circular disc centered at $\underline{x}$ of radius $\delta$; Figure 1 helps to elucidate this concept.

Further, the pairwise force function $f$ represents the force between two particles separated by a distance of length $\xi$ [Silling 2000]. Thus it must exhibit the properties

$$
\begin{gathered}
f(-\underline{\eta},-\underline{\xi})=-f(\underline{\eta}, \underline{\xi}), \\
(\underline{\xi}+\underline{\eta}) \otimes f(\underline{\eta}, \underline{\xi})=0,
\end{gathered}
$$

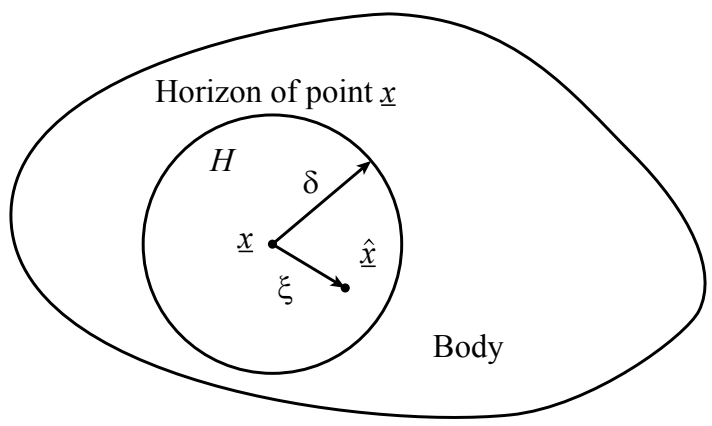

Figure 1. Each point $\underline{x}$ in the body $\Omega$ interacts directly with the points $\underline{\hat{x}}$ in the circular disc $H(\underline{x})$ through bonds. 
where $\otimes$ is the symbol of the tensor product, and the vector $\eta$ is defined by the equation

$$
\underline{\eta}=\left[\begin{array}{l}
u(\underline{x})-u(\underline{\hat{x}}) \\
v(\underline{x})-v(\underline{\hat{x}})
\end{array}\right] .
$$

These restrictions ensure the conservation of angular momentum and the collinearity of the force with respect to the relative position of the particles. Details on the force function can be found in [Silling 2000]. Next, for the linearized pairwise force function introduced in that reference, the force yields

$$
f(\underline{\eta}, \underline{\xi})=C(\underline{\xi}) \underline{\eta}
$$

where the micromodulus $C$ satisfies the condition

$$
C(-\underline{\xi})=C(\underline{\xi}) .
$$

A fundamental measure of peridynamic theory is the bond stretch given by the formula

$$
s=\frac{|\underline{\xi}+\underline{\eta}|-|\underline{\xi}|}{|\underline{\xi}|} .
$$

Specifically, stretch is used to determine whether the bond has failed or not, and thus it is the measure governing the force between the particles, like strain in classical mechanics theory. Bonds which have exceeded the predetermined value of $s_{0}$ are damaged. In quantifying the damage on a specific point $\underline{x}$ from the points in the horizon of $\underline{x}$ the function

$$
\phi(\underline{x}, t)=1-\frac{\int_{H(\underline{x})} \mu(\underline{x}, t, \underline{\xi}) d V_{\underline{\hat{x}}}}{\int_{H(\underline{x})} d V_{\underline{\hat{x}}}}
$$

is used, where the damage can be assumed to cause total failure of the bond by

$$
\mu(\underline{\xi}, t)= \begin{cases}1 & \text { if } s(t, \underline{\xi})<s_{0} \\ 0 & \text { otherwise }\end{cases}
$$

In linear peridynamics, the pairwise force amplitude is given by the equation [Silling 2000; Silling and Askari 2005]

$$
|f(\underline{\eta}, \underline{\xi})|=c \frac{|\underline{\eta}|}{|\underline{\xi}|},
$$

where the force vector $f$ is aligned with the vector $\underline{\xi}+\underline{\eta}$, and for a 2D plate problem the constant $c$ is given by

$$
c=\frac{9 E}{2 \pi \delta^{3}},
$$

where $k$ is the bulk modulus of the material in [Emmrich and Weckner 2007a]. Several other formulations can be found in [Silling 2000] regarding the pairwise force function. 
2.2. Polar coordinate transformation. Equation (1) can also be cast into polar coordinates, yielding

$$
\rho\left[\begin{array}{l}
\ddot{u}(\underline{x}, t) \\
\ddot{v}(\underline{x}, t)
\end{array}\right]+\int_{H(\theta, r)} f\left(\left[\begin{array}{c}
u(\underline{x}, t)-u(\underline{x}+\underline{r}, t) \\
v(\underline{x}, t)-v(\underline{x}+\underline{r}, t)
\end{array}\right],-\underline{r}\right)|J| d \theta d r=\left[\begin{array}{l}
b_{x}(\underline{x}, t) \\
b_{y}(\underline{x}, t)
\end{array}\right],
$$

where

$$
\begin{aligned}
|J| & =-r, \\
\underline{r} & =-\underline{\xi}, \\
\underline{r} & =r\left(\begin{array}{l}
\cos \theta \\
\sin \theta
\end{array}\right) .
\end{aligned}
$$

Note that (9) in polar coordinates yields

$$
\underline{\eta}=\left[\begin{array}{l}
u(\underline{x})-u(\underline{x}+\underline{r}) \\
v(\underline{x})-v(\underline{x}+\underline{r})
\end{array}\right]
$$

and equivalently (15) yields

$$
|f(\underline{\eta}, \underline{r})|=\frac{c}{r}|\underline{\eta}|
$$

For purposes of elucidation, the displacements $u$ and $v$ of a bond are shown in Figure 2.

Adopting the linearized pairwise force function, and using the fact that the horizon $\delta$ is a small distance, the total stretch of the bond can be approximated quite accurately by just the collinear component of the vector $r$. The contribution to the extension of the bond from the perpendicular displacements with respect to the vector $\underline{r}$ shown in Figure 2 can be neglected for small distances $r$. This is due to the assumption that small rigid body rotations of the bond can be neglected (see Appendix C). With this assumption and since this force is in the direction of the vector $\underline{r}$ the forces per unit volume in the $x$ and $y$ directions

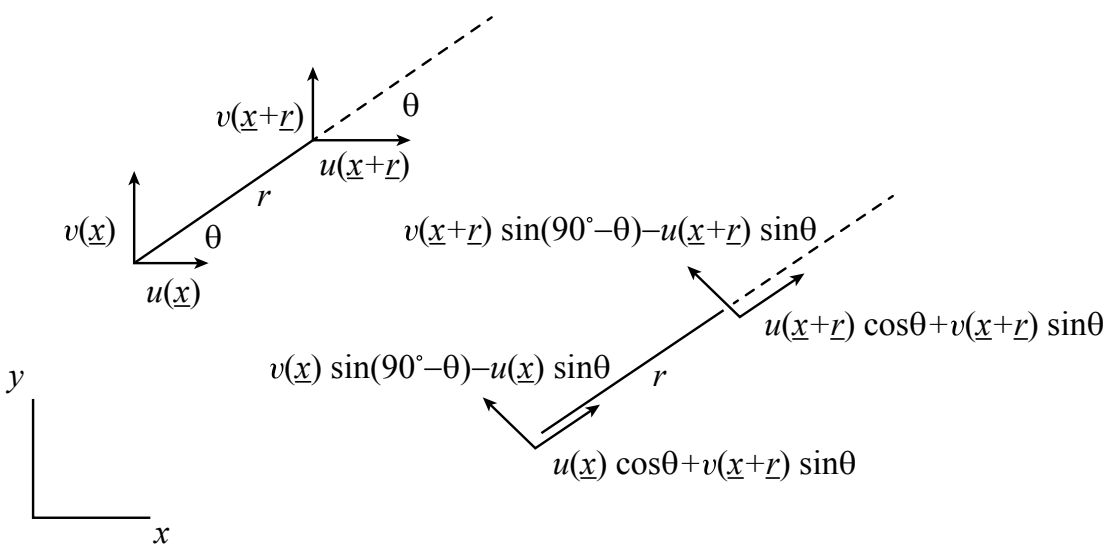

Figure 2. A bond of length $r$ and angle $\theta$ with respect to the global coordinate system. 
yield

$$
\begin{aligned}
& \text { force }_{x}=\frac{c}{r}\left((u(\underline{x})-u(\underline{x}+\underline{r})) d V_{\underline{x}+\underline{r}} \cos \theta+(v(\underline{x})-v(\underline{x}+\underline{r})) d V_{\underline{x}+\underline{r}} \sin \theta\right) \cos \theta, \\
& \text { force }_{y}=\frac{c}{r}\left((v(\underline{x})-v(\underline{x}+\underline{r})) d V_{\underline{x}+\underline{r}} \cos \theta+(v(\underline{x})-v(\underline{x}+\underline{r})) d V_{\underline{x}+\underline{r}} \sin \theta\right) \sin \theta .
\end{aligned}
$$

These equations represent the force in the $x$ and $y$ directions due to one bond formed from two particles at distance $r$ and at angle from the reference point $\theta$. Since (17) is given in polar coordinates, (1) can be cast as

$$
\rho\left[\begin{array}{l}
\ddot{u}(\underline{x}, t) \\
\ddot{v}(\underline{x}, t)
\end{array}\right]+\int_{0}^{\delta} \int_{0}^{2 \pi} \frac{c}{r}\left[\begin{array}{cc}
\cos ^{2} \theta & \cos \theta \sin \theta \\
\cos \theta \sin \theta & \sin ^{2} \theta
\end{array}\right]\left(\begin{array}{l}
u(\underline{x}, t)-u(\underline{x}+\underline{r}, t) \\
v(\underline{x}, t)-v(\underline{x}+\underline{r}, t)
\end{array}\right)|J| d \theta d r=\left[\begin{array}{l}
b_{x}(\underline{x}, t) \\
b_{y}(\underline{x}, t)
\end{array}\right] .
$$

\section{Spatial discretization}

3.1. Radial basis function expansion. Having derived (24), we proceed to use the Kansa collocation method for its spatial discretization; see [Kansa 1990a; 1990b]. In implementing the Kansa collocation method, a series of nodes is distributed in the domain forming a grid of points. Inverse multiquadric (IMQ) radial basis functions (RBFs) are used as defined in those references; they correspond to the inverse of the Euclidean distance of the point $\underline{x}$ from the collocation node $k$ :

$$
g_{k}(\vec{x})=\frac{1}{\sqrt{\left(x-x_{k}\right)^{2}+\left(y-y_{k}\right)^{2}+\psi_{k}^{2}}},
$$

where the distance $\psi_{k}$ is a local shape parameter regulating the shape of the basis. Large values of this parameter contribute to smoother shapes and are quite accurate approximations of flat and slowly varying solutions. However, small parameters represent sharper shapes and are particularly good for peaks and steep slopes. The IMQ function attains its maximum at the node and monotonically decreases as the distance from the node increases. Next, using the same IMQs for both the $u$ and $v$ displacements yields

$$
u(\underline{x})=\sum_{k=1}^{N} a_{1 k} g_{k}(\underline{x})+\sum_{j=1}^{M} a_{2 j} q_{j}(\underline{x}), \quad v(\underline{x})=\sum_{k=1}^{N} d_{1 k} g_{k}(\underline{x})+\sum_{j=1}^{M} d_{2 j} q_{j}(\underline{x}) .
$$

Further, the polynomials $q$ that correspond to the $a$ and $d$ coefficients can be arbitrarily chosen. Note that details on the Kansa collocation method and the IMQ RBFs are included in Appendix B. Next, (26) on the collocation points with time-dependent coefficients can be cast in the form

$$
\left[\begin{array}{c}
u\left(\underline{x}_{1}, t\right) \\
v\left(\underline{x}_{1}, t\right) \\
\vdots \\
0 \\
\vdots
\end{array}\right]=\left[\begin{array}{cccccc}
g_{1}\left(\underline{x}_{1}\right) & 0 & \ldots & q_{1}\left(\underline{x}_{1}\right) & 0 & \ldots \\
0 & g_{1}\left(\underline{x}_{1}\right) & \ldots & 0 & q_{1}\left(\underline{x}_{1}\right) & \ldots \\
\vdots & \vdots & \vdots & \vdots & \vdots & \vdots \\
q_{1}\left(\underline{x}_{1}\right) & q_{1}\left(\underline{x}_{2}\right) & q_{1}\left(\underline{x}_{3}\right) & \ldots & 0 & 0 \\
\vdots & \vdots & \vdots & \vdots & \vdots & \ddots
\end{array}\right]\left[\begin{array}{c}
a_{11}(t) \\
d_{11}(t) \\
\vdots \\
a_{21}(t) \\
d_{21}(t) \\
\vdots
\end{array}\right] .
$$

Equation (27) is the basis upon which the 2D solution of the integrodifferential equation is expanded. The difference in the displacements of a bond, denoted by the vector $\eta$ and shown in (21), is obtained 
using (26). Specifically, the vector $\eta$ with time dependence yields

$$
\underline{\eta}(\underline{x}, \underline{r}, t)=\left[\begin{array}{ccccccc}
G_{1}(\underline{x}, \underline{r}) & 0 & G_{2}(\underline{x}, \underline{r}) & \ldots & Q_{1}(\underline{x}, \underline{r}) & 0 & \ldots \\
0 & G_{1}(\underline{x}, \underline{r}) & 0 & \ldots & 0 & Q_{1}(\underline{x}, \underline{r}) & \ldots
\end{array}\right]\left[\begin{array}{c}
a_{11}(t) \\
d_{11}(t) \\
\vdots \\
a_{21}(t) \\
d_{21}(t) \\
\vdots
\end{array}\right],
$$

where

$$
G_{k}(\underline{x}, \underline{r})=g_{k}(\underline{x})-g_{k}(\underline{x}+\underline{r}), \quad Q_{j}(\underline{x}, \underline{r})=q_{j}(\underline{x})-q_{j}(\underline{x}+\underline{r}) .
$$

3.2. Stiffness determination. Having represented the displacements by linear combinations of the basis functions, an approach similar to the one of FEM is followed. It is assumed that the displacement of any given point inside the domain is captured by interpolating the four adjacent nodes surrounding the point. Figure 3, left, elucidates this interpolation scheme.

It is clear that for any point that belongs in the square, the four adjacent nodes contribute to its displacement. This leads to a quite convenient and efficient way to integrate over the horizon of each node. Figure 3, right, shows the horizon of each node in a specific element.

Obviously the integration of (1) can be performed using a finite element approximation inside an element. Specifically, integration over the horizon is required only for one element and its four nodes. Then, the integration over the horizon of each node is performed by merely adding the nodal values of the elements, identically as done in FEM. Therefore combining (21), (24), and (28), the peridynamic stiffness density of the node $i$ is given as

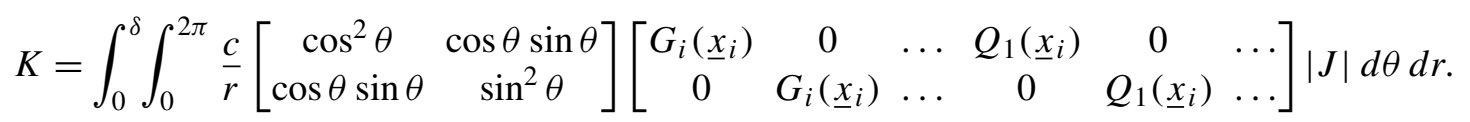

In this regard, combining (30) with (18) yields the second-order micromodulus tensor

$$
C(\underline{\xi})=c\left[\begin{array}{cc}
\cos ^{2} \theta & \cos \theta \sin \theta \\
\cos \theta \sin \theta & \sin ^{2} \theta
\end{array}\right]
$$
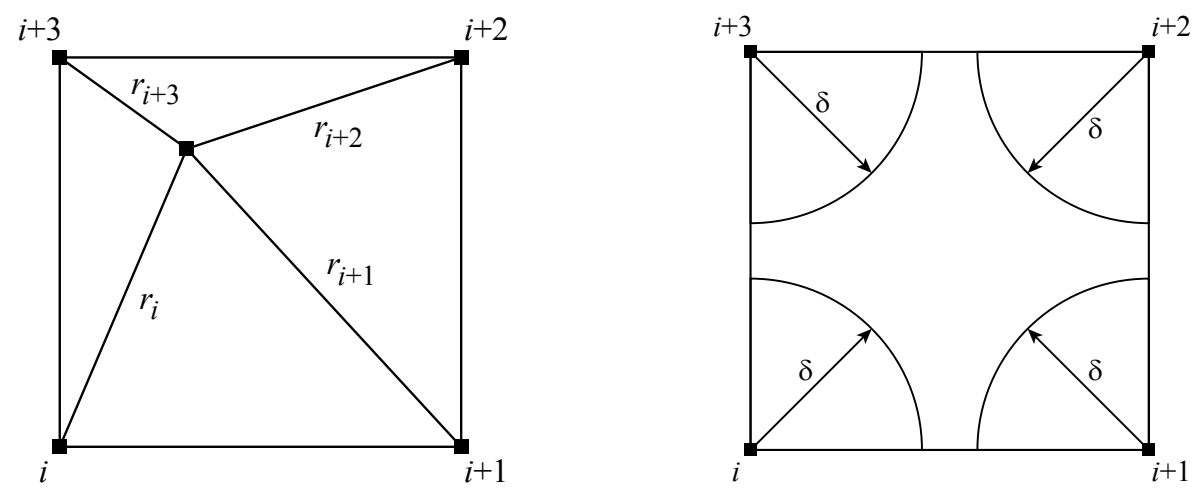

Figure 3. Left: a point of interest surrounded by its four closest nodes. Right: horizon of each node in a square element formed by four grid points. 
and combining (4), (19), and (20), (31) can be cast in a form identical to that of [Silling 2000] as

$$
C(\underline{\xi})=\frac{c}{|\underline{\xi}|^{2}} \underline{\xi} \otimes \underline{\xi} .
$$

Next, the stiffness density matrix corresponding to the $g$ basis functions for a four-node element consists of $4 \times 4$ blocks of $2 \times 2$ matrices and yields

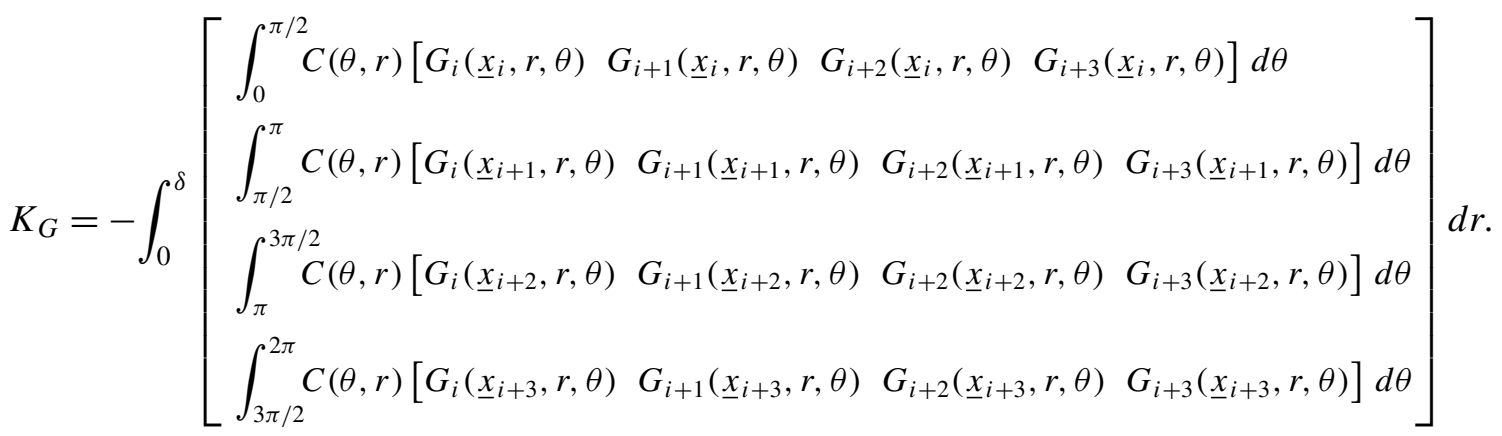

Further, the stiffness density matrix corresponding to the $q$ basis functions for the node $i$ consists of a $2 \times M$ matrix, where $M$ is the number of the $q$ functions, and yields

$$
K_{Q}=-\int_{0}^{\delta}\left[\int_{0}^{2 \pi} C(\theta, r)\left[Q_{1}\left(\underline{x}_{i}, r, \theta\right) d \theta \ldots Q_{M}\left(\underline{x}_{i}, r, \theta\right)\right] d \theta\right] d r .
$$

\section{Implementation aspect}

4.1. Linear differential equations. Utilizing the concept shown in Figure 3 and combining (1), (33), and (34) yields a set of linear differential equations. Specifically, the set of linear second-order differential equations can be cast in the matrix form as

$$
\left[\begin{array}{cc}
\sum_{i} \rho_{i} G_{i} & \sum_{j} \rho_{j} Q_{j} \\
\sum_{j} Q_{j}^{T} & 0
\end{array}\right]\left[\begin{array}{c}
a_{11} \\
d_{11} \\
\vdots \\
a_{21} \\
d_{21} \\
\vdots
\end{array}\right]+\left[\begin{array}{cc}
\sum_{i} K_{G i} & \sum_{j} K_{Q i} \\
\sum_{j} Q_{j}^{T} & 0
\end{array}\right]\left[\begin{array}{c}
a_{11} \\
d_{11} \\
\vdots \\
a_{21} \\
d_{21} \\
\vdots
\end{array}\right]=\left[\begin{array}{c}
b \\
0
\end{array}\right]
$$

where $\sum_{i} \rho_{i} G_{i}$ is a $2 N \times 2 N$ square matrix representing the nodal material density related to the $g$ basis functions, $\sum_{j} \rho_{j} Q_{j}$ is a $2 N \times 2 M$ rectangular matrix representing the nodal material density related to the additional basis functions $q, \sum_{j} Q_{j}^{T}$ is a $2 M \times 2 N$ rectangular matrix and is called the regularization condition, and finally 0 is a $2 M \times 2 M$ zero matrix. More details on the regularization conditions can be found in [Kansa 1990a; 1990b] and in Appendix B. Equivalently $\sum_{i} K_{G i}$, which is a square $2 N \times 2 N$ matrix, is the stiffness density related to the $g$ basis functions, $\sum_{j} K_{Q i}$ is the $2 N \times 2 M$ stiffness density matrix related to the $q$ basis functions, and $\left[\begin{array}{l}b \\ 0\end{array}\right]$ is the $2 N \times 1$ loading vector on top of a $2 M \times 1$ zero vector representing the regularization conditions. Further, $(35)$ is a $2(N+M) \times 2(N+M)$ system of second-order linear differential equations and can be readily integrated in time using a time 
integration scheme. Note that since the quantities at (30), (33), and (34) represent the stiffness density, this collocation approach is different from FEM.

4.2. Crack-inclusion treatment. Next, we proceed to address the crack growth or crack initiation problem utilizing the preceding peridynamic theory. In this regard, assume that a crack exists in a specific position in the medium. With the above formulation this crack will affect only the peridynamic stiffness of the surrounding nodes. Naturally the cracks interrupt the horizon of the nodes surrounding them; Figure 4 shows how the horizon of node $i+1$ is affected.

It is perhaps easier to appreciate from Figure 4 how powerful the peridynamic formulation becomes when it comes to dealing with discontinuities in the domain. The crack essentially describes the bond failure at these points and therefore node $i+1$ along with the other nodes cannot "see" past the crack, thus inducing less stiffness density. This is implemented by using the same equations as before, only with different integration limits in (33) and (34). The integration over the discontinuous horizon poses many difficulties; it is carried out numerically in Appendix A. Gaussian quadrature is used for the element $K_{G}$ referring to the healthy part of the domain, shown in Appendix A. Despite the fact that a more laborious numerical integration is needed for the cracked element, peridynamic modeling through this approach is quite efficient since a uniform grid of points would have only one kind of element and thus no other integration is needed. For the integration over the horizon of the functions $Q$, closed-form solutions are available for "healthy" bonds around the nodes, but for discontinuities numerical integration is again needed, as shown in Appendix A.

Keeping in mind that the collocation approach yields stiffness density, in contrast to the finite element formulation, which yields stiffness, note that the weak form of the problem, given in [Emmrich and Weckner 2007b], is

$$
\begin{aligned}
& \int_{R} \rho(\underline{x}) \partial_{t}^{2}\left(\begin{array}{c}
u(\underline{x}) \\
v(\underline{x})
\end{array}\right) w(\underline{x}) d V_{\underline{x}}+\frac{1}{2} \int_{R} \int_{H(\underline{x})}\left(\begin{array}{c}
u(\underline{\hat{x}}) \\
v(\underline{\hat{x}})
\end{array}\right) C(\underline{x}, \underline{\hat{x}}) w(\underline{x}) d V_{\underline{x}} d V_{\underline{\hat{x}}} \\
& =\int_{R}\left(\begin{array}{l}
b_{x}(\underline{x}, t) \\
b_{y}(\underline{x}, t)
\end{array}\right) w(\underline{x}) d V_{\underline{x}} .
\end{aligned}
$$

In this equation $w$ denotes the weight function, and substituting the displacement function in (36) yields the Galerkin approximation, which leads to FEM. As is clear for the stiffness expression, a double

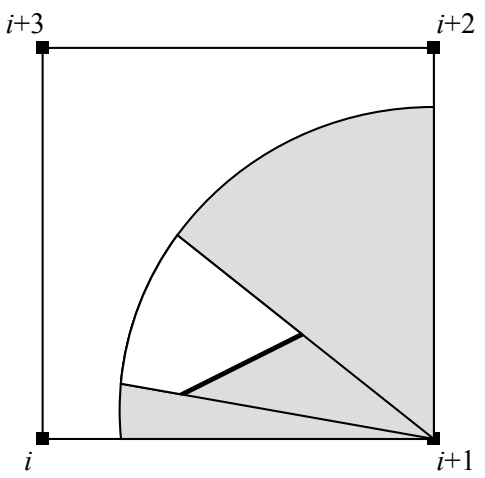

Figure 4. The disrupted horizon of node $i+1$, as it is modeled in peridynamics. 


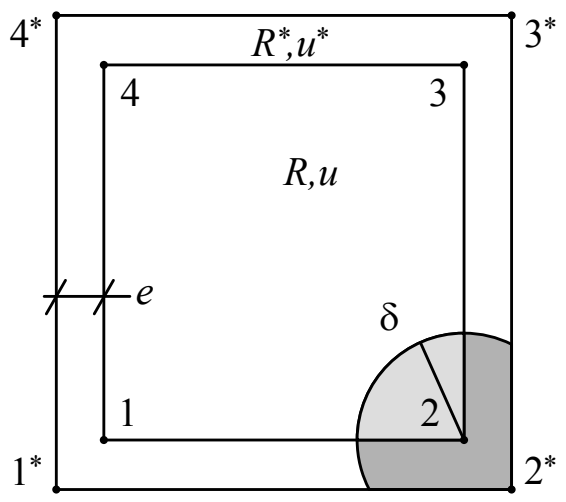

Figure 5. The additional layer of material $R^{*}$ needed for implementing the boundary conditions.

integration is involved, one on the domain $R$ and one in the horizon domain $H$. This emphasizes that in the formulation of (33) and (34), the stiffness density at a collocation point is calculated. Therefore (1) is solved not in terms of displacements and nodal forces as it is from (36), but rather in terms of loading density at the collocation points and displacements. Utilizing this formulation, the peridynamic boundary conditions are applied in a much simpler way since the input loading is actually a load density. Further, two consecutive integrations over the discontinuous domains $R$ and $H$ are quite laborious. This is in fact the reason why the finite element formulation is substituting the large number of bonds in an equivalently large number of truss elements in [Macek and Silling 2007]. The formulation proposed herein is quite similar to the truss approach; each node is virtually connected to all the trusses inside its horizon and its stiffness is obtained by direct integration without the actual introduction of the trusses. Further, since the crack is handled geometrically inside the element, there is no need of checking all the bonds inside the domain. The displacements inside the domain are interpolated from the nodal displacements and are directly obtained. Then, the energy release rate is calculated around the crack independently from the mesh size. Naturally, for a more accurate approximation around the crack tip, enrichment of the $q$ functions according to [Fleming et al. 1997] is available in addition to the grid refinement.

4.3. Peridynamic boundary conditions. Having derived the governing equation as a set of linear secondorder differential equations in matrix form, we proceed to incorporate the boundary conditions. Details on incorporating boundary conditions in peridynamic theory are covered in [Silling 2000]. However, due to this formulation the implementation of boundary conditions becomes readily available. Specifically, an additional layer of material is added to the external part of the elements standing on the boundaries of the domain; it is denoted by $R^{*}$ in [Silling 2000]. The corresponding displacements of the additional layer are decomposed on the same $g$ and $q$ basis functions and therefore the displacements of the material $R^{*}$, denoted by $u^{*}$ in [Silling 2000], are given from (26). Figure 5 shows the additional layer of material $R^{*}$ that needs to be added on the boundaries.

Next, the stiffness density related to the lightly shaded area has been already calculated from the preceding equations. However, the stiffness density related to a small layer of thickness $e$ and shown in Figure 5 with the darker shade needs to be added to the stiffness density of the nodes. In this regard, since the displacements $u^{*}$ are decomposed into the same basis as the displacements $u$, the boundary 
conditions, such as simple supports, etc., can be applied on the external nodes $1^{*}, 2^{*}$, etc., by utilizing the expansion basis and the displacements of these nodes. Next, for imposing certain boundary conditions on the collocation nodes of the Kansa method, specific rows in the matrices shown in (35) are changed to account for the imposed constraints. Details can be found on the implementation of boundary conditions of the Kansa collocation method in [Kansa 1990a; 1990b]. Note that the governing equation is solved in terms of displacements $u(x)$ and $v(x)$ and force density $b(x)$, and since the force loading conditions by definition are force densities, the boundary conditions are imposed readily using the basis functions. For comparison with any theoretical models involving stress applied on the above described example, the loading density $b(x)$ must be multiplied by the layer thickness $e$ to yield force per unit area.

\section{Illustrative example}

To illustrate the applicability of the proposed approach, a deterministic problem is considered. In this regard, a square 2D plate of dimensions $1 \mathrm{~cm} \times 1 \mathrm{~cm}$ and bulk modulus $k=1 \mathrm{~N} / \mathrm{cm}^{2}$ under tensile stress is modeled with FEM and peridynamic theory. The tension is of unitary amplitude $\sigma=1 \mathrm{~N} / \mathrm{cm}^{2}$ and the displacements obtained by peridynamic theory are compared to the FEM displacements. Figure 6 shows the equivalent modeling of this simple problem with FEM and the proposed peridynamic theory involving the Kansa collocation method.

For FEM, the nodal forces are readily calculated using the stress applied on that edge. In peridynamic theory, however, the nodal forces are force densities and $b^{*} e=\sigma$ thus the force density simply depends on the additional layer thickness. The displacements given by the finite element model are compared to the ones obtained from the proposed formulation in Table 1 on the next page; a layer of thickness $e=\delta / 5$ is assumed for the numerical calculations.

Next, a cracked element is considered. Figure 7 shows a simple patch test used to verify displacements for a cracked square plate under tension.
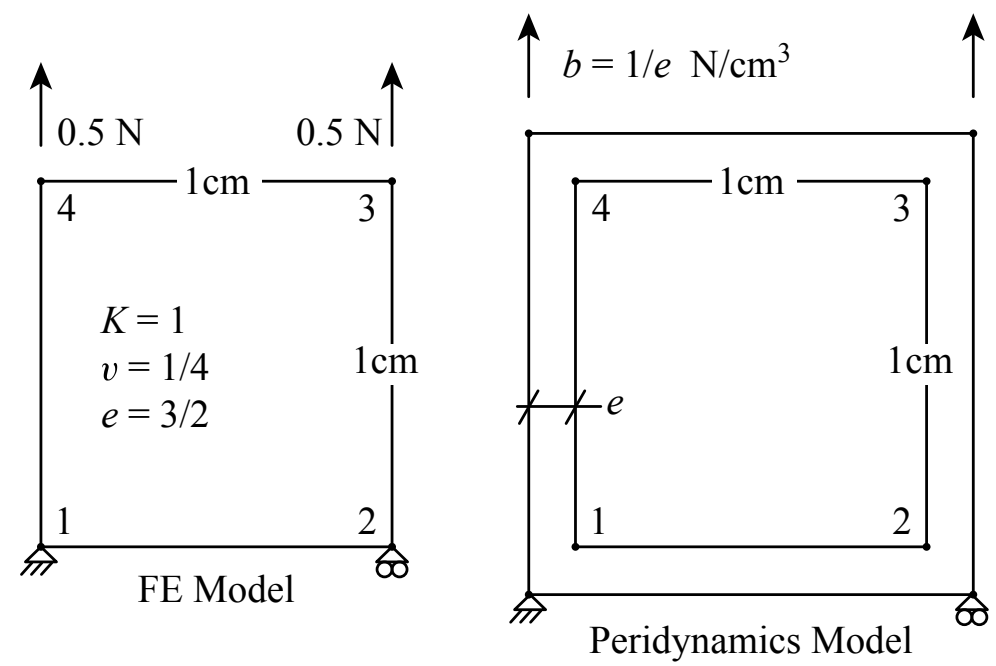

Figure 6. A simple 2D plate of unit thickness under tension of $\sigma=1 \mathrm{~N} / \mathrm{cm}^{2}$, modeled via FEM and peridynamic theory by the Kansa collocation method. 


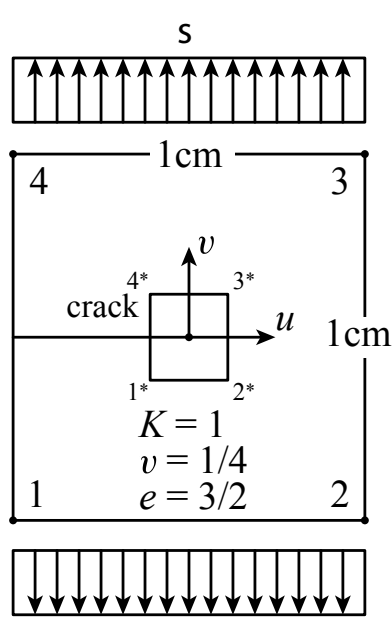

Theoretical Model

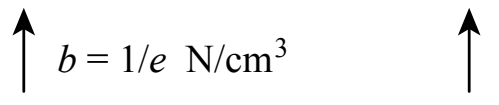

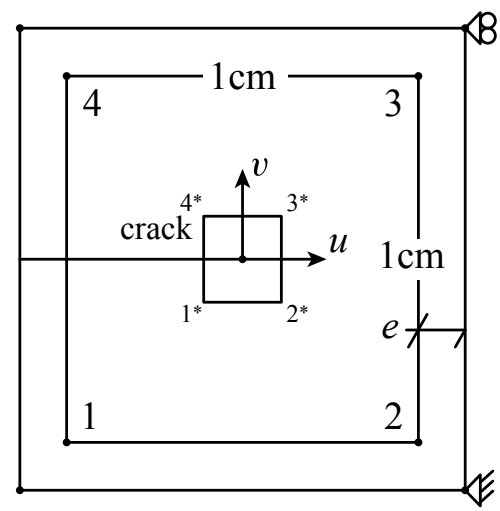

Peridynamics Model

Figure 7. A cracked square plate under uniform tension modeled via peridynamic theory and the Kansa collocation method.

The displacements of the theoretical solution are given by the closed-form equations in [Gdoutos 1990] and yield

$$
u(\underline{x})=\frac{k_{1}}{2 \mu} \sqrt{\frac{r}{2 \pi}} \cos \left(\frac{\theta}{2}\right)\left[k_{2}-1+2 \sin \left(\frac{\theta}{2}\right)\right], \quad v(\underline{x})=\frac{k_{1}}{2 \mu} \sqrt{\frac{r}{2 \pi}} \sin \left(\frac{\theta}{2}\right)\left[k_{2}+1-2 \cos \left(\frac{\theta}{2}\right)\right] .
$$

Taking into account the symmetry of the loading, and transforming the coordinate system, the displacements of the two models are compared for the displacements $u$ and $v$. The symbol $k_{1}$ stands for the first mode stress intensity factor, $r$ is the distance from the center of the element, $\mu$ stands for the shear modulus, $\theta$ is the angle with respect to the initial crack, and $k_{2}$ is a parameter depending on the Poisson ratio and the crack geometry. For the element in Figure 7 the theoretical displacements are compared with the results of the peridynamic formulation. However, the peridynamic results with this formulation were not accurate. A more accurate approximation of the displacements around the crack tip points is achieved through the enrichment of the basis functions $q$ from [Fleming et al. 1997] with the basis

$$
q_{5}=\sqrt{r} \cos \frac{\theta}{2}, \quad q_{6}=\sqrt{r} \sin \frac{\theta}{2}, \quad q_{7}=\sqrt{r} \sin \frac{\theta}{2} \sin \theta, \quad q_{8}=\sqrt{r} \cos \frac{\theta}{2} \sin \theta .
$$

\begin{tabular}{|ccccc|ccccc|}
\hline \multicolumn{3}{|c|}{ Peridynamics via Kansa collocation method } & \multicolumn{5}{|c|}{ FEM displacements } \\
Node & $u(\mathrm{~cm})$ & $v(\mathrm{~cm})$ & $\epsilon_{x}$ & $v$ & Node & $u(\mathrm{~cm})$ & $v(\mathrm{~cm})$ & $\epsilon_{x}$ & $v$ \\
1 & -0.027 & 0.112 & -0.160 & 0.237 & 1 & 0 & 0 & -0.167 & 0.250 \\
2 & -0.187 & 0.112 & & & 2 & -0.167 & 0 & & \\
3 & -0.026 & 0.787 & $\epsilon_{y}$ & & 3 & 0 & 0.667 & $\epsilon_{y}$ \\
4 & -0.187 & 0.787 & 0.674 & & 4 & -0.167 & 0.667 & 0.667 \\
\hline
\end{tabular}

Table 1. Comparison of displacements for a plate under uniform tension. 


\begin{tabular}{|ccc|ccr|}
\hline \multirow{2}{*}{$\begin{array}{c}\text { Peridynamics } \\
\text { Node* }\end{array}$} & $u(\mathrm{~cm})$ & $v(\mathrm{~cm})$ & \multicolumn{3}{|c|}{ Theoretical displacements } \\
1 & 0.128 & -0.31 & 1 & 0.1354 & -0.3269 \\
2 & 0.179 & -0.078 & 2 & 0.1706 & -0.0707 \\
3 & 0.178 & 0.079 & 3 & 0.1706 & 0.0707 \\
4 & 0.126 & 0.31 & 4 & 0.1354 & 0.3269 \\
\hline
\end{tabular}

Table 2. Comparison of displacements for a cracked plate under uniform tension.

The problem of obtaining the displacements around the crack tip was encountered in [Fleming et al. 1997] and the results were accurate enough when the basis was expanded with the basis containing the displacements near the crack tip. Thus, the linear functions $q$ are enriched with an additional four basis functions for the crack tip. For the simple example shown in Figure 7, Table 2 summarizes the results for the corners of the square inside the element with side length $0.2 \mathrm{~cm}$.

The numerical results obtained prior to the basis enrichment are omitted due to their poor accuracy. However, it has been seen that the numerical results after the enrichment are significantly better than the ones obtained without the basis enrichment. Further, there are persistent errors which must be addressed. The horizon $\delta$ that has been used in both examples is $\delta=1 \mathrm{~cm}$ and the solution of the first example remains constant for any $\delta$ value less than $1 \mathrm{~cm}$. The displacements obtained from the cracked domain appear to be dependent on the horizon length for values larger than $\delta=0.80 \mathrm{~cm}$, which is another issue that needs to be addressed. Integration over the horizon for the IMQ basis functions is achieved by implementing the "visibility" criterion, extensively described in [Fleming et al. 1997]. That is, the crack interrupts the horizon and thus the values of the $g$ basis functions beyond the crack line are set equal to zero. The same concept holds for all the $q$ basis functions.

\section{Stochastic peridynamic theory}

6.1. Maximum energy release rate criterion. The reliability of a structure including a crack or initiating a crack is considered in this section. Specifically, the probability of crack propagation and the direction in which the crack will propagate is of particular interest. For this, the position of the propagating or initiating fracture can be determined by the maximum energy release rate for brittle materials in [Gdoutos 1990]. To obtain the energy release rate, the length of the crack propagation is preselected as $\delta \alpha$ and therefore points to be checked lay on the circular area given by the selected equation

$$
\underline{x}_{\text {test }}=\underline{x}_{c}+\delta \alpha\left(\begin{array}{c}
\cos \phi \\
\sin \phi
\end{array}\right)
$$

for $\phi \in[0,2 \pi]$, where $\underline{x}_{\text {test }}$ is the position of the potential next crack tip and $\underline{x}_{c}$ is the position of the current crack tip. Since the points on the circle $\underline{x}_{\text {test }}$ and the crack tip $\underline{x}_{c}$ form potential fracture surfaces, the work of all the tensile bonds per unit area of potential fracture can be directly calculated by (see [Silling and Askari 2005])

$$
G_{E}=\int_{z} \int_{V^{\prime}} w(\underline{\eta}, \underline{\xi}) d V^{\prime} d z
$$




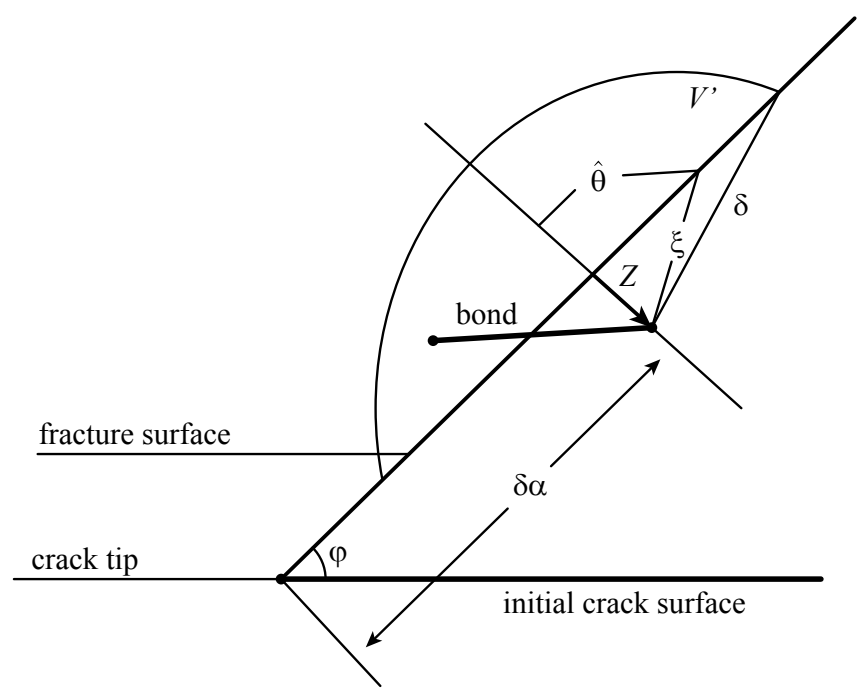

Figure 8. The propagating fracture of length $\delta \alpha$ at angle $\phi$ from the initial crack and the calculation of the energy release rate per unit of fractured area.

In this equation $z$ denotes the perpendicular distance of a point from the fractured surface and $V^{\prime}$ is the volume of integration. Figure 8 helps to elucidate the concept of the distance $z$ and the volume of integration. For the linearized pairwise force function, the work of a single bond yields

$$
w(\underline{\eta}, \underline{\xi})=\frac{1}{2} c s^{2}|\underline{\xi}|,
$$

and utilizing (19) yields the energy per unit surface area of a 2D plate with unit thickness:

$$
G_{E}=\int_{0}^{\delta} \int_{z}^{\delta} \int_{-\cos ^{-1}(z / r)}^{\cos ^{-1}(z / r)} \frac{1}{2} c s^{2} r^{2} d \hat{\theta} d r d z
$$

Figure 8 also helps visualize the concept of bonds breaking and forming a cracked surface. Obviously, the energy per unit surface area depends on the initial crack tip, the angle $\phi$, and the length $\delta \alpha$, since the stretch $s$ depends on these parameters. From the assumption of a linear pairwise force function and small rigid body rotations shown in Appendix $\mathrm{C}$, the stretch of the bonds can be calculated from a simpler form than (12). This form is

$$
s(\underline{x}, r, \theta)=\frac{1}{r}\left[\begin{array}{ll}
\cos \theta & \sin \theta
\end{array}\right]\left[\begin{array}{c}
u(\underline{x})-u(\underline{x}+\underline{r}) \\
v(\underline{x})-v(\underline{x}+\underline{r})
\end{array}\right],
$$

and Figure 2 helps to illustrate this concept. Next, combining (43), (42), and (39) and correlating the angles $\hat{\theta}, \phi$, and $\theta$ through the simple equation

$$
\pi / 2-\hat{\theta}+\phi=\theta
$$

yields

$$
G_{E}\left(\delta \alpha, \phi, \underline{x}_{c}\right)=\int_{0}^{\delta} \int_{z}^{\delta} \int_{\phi+\sin ^{-1}(z / r)}^{\phi+\pi-\sin ^{-1}(z / r)}\left|\underline{\eta}\left(\theta, \xi, z, \delta \alpha, \phi, \underline{x}_{c}\right)\right|^{2} d \theta d r d z
$$


where $|\eta|$ is given for the linearized pairwise force function shown in Appendix $\mathrm{C}$ as

$$
|\underline{\eta}|=s|\underline{\xi}| \text {. }
$$

Naturally, the angle at which the tensile energy per unit area is the maximum can be chosen as the fracture propagation angle; see [Gdoutos 1990]. Further, in brittle materials the energy release is a measurable quantity and can be used in real applications as a threshold for fracture. Figure 8 can be used to clarify this concept; see also [Silling and Askari 2005].

6.2. Stochastic fracture on elastostatic peridynamic theory. Having derived the energy release rate as a function of the crack tip $\underline{x}_{c}$, propagating crack length $\delta \alpha$, and propagating crack angle $\phi$, we proceed to probabilistically identify the propagation angle. In this context, in classical mechanics, materials with random properties are modeled by expressing the Young's modulus as a random process. However, in the peridynamic approach this must be modified. The fact that each node is connected with an infinite number of points belonging in the node's horizon should make the random process which represents the micromodulus of the bonds depend both on the angle $\theta$ and on the distance $r$ in the polar coordinate system. In this paper, the bond micromodulus is treated as a random process depending only on the distance from the node $r$. Therefore, the micromodulus coefficient $c$ is a random process $c(r, \vartheta)$ where $\vartheta$ is the random parameter. Further, the loading of the structure involves uncertainties which are specified in terms of a random variable. The PDF of the energy release rate $G_{E}$ given in (45) must be calculated in order for the reliability of the crack propagation to be calculated. Naturally the PDF of the coefficients for the static case can be obtained by utilizing (35), which yields

$$
\left[\begin{array}{c}
a_{11} \\
d_{11} \\
\vdots \\
a_{21} \\
d_{21} \\
\vdots
\end{array}\right]=\left[\begin{array}{cc}
\sum_{i} K_{G i} & \sum_{j} K_{Q i} \\
\sum_{j} Q_{j}^{T} & 0
\end{array}\right]^{-1}\left[\begin{array}{l}
b \\
0
\end{array}\right] .
$$

Obviously, the equation above includes a random matrix inversion and a multiplication by a random forcing vector. In this context, (46) combined with (43) can be cast in the form

$$
|\underline{\eta}|=\sum_{i=1}^{n} \alpha_{i} \beta_{i}
$$

where $\alpha_{i}=\left[\begin{array}{lll}a & \ldots & d\end{array}\right]$ is the set of the random coefficients, and

$$
\beta_{i}=\left[\begin{array}{llll}
G_{1}\left(\underline{x}_{\text {test }}+\underline{z}, \underline{r}\right) \cos \theta \quad \ldots \quad Q_{4}\left(\underline{x}_{\text {test }}+\underline{z}, \underline{r}\right) \sin \theta
\end{array}\right]
$$

is the set of the deterministic functions which depend on the angle $\phi$ and the length $\delta \alpha$. Clearly,

$$
|\underline{\eta}|^{2}=\sum_{i=1}^{n}\left(\alpha_{i} \beta_{i}\right)^{2}+2 \sum_{i=1}^{n-1} \sum_{l}^{n} \alpha_{i} \alpha_{l} \beta_{i} \beta_{l},
$$


where $l>i$. In this case, the PDF of the energy release at a given orientation can be determined by combining (49) and (45) and yields

$$
\begin{aligned}
G_{E}\left(\delta \alpha, \phi, x_{c}\right)= & \int_{0}^{\delta} \int_{z}^{\delta} \int_{\phi+\sin ^{-1}(z / r)}^{\phi+\pi-\sin ^{-1}(z / r)}\left[\beta_{1}(\theta, r, z)^{2} \ldots \beta_{n}(\theta, r, z)^{2}\right] d \theta d r d z\left[\begin{array}{c}
\alpha_{1}^{2} \\
\vdots \\
\alpha_{n}^{2}
\end{array}\right] \\
& +2 \int_{0}^{\delta} \int_{z}^{\delta} \int_{\phi+\sin ^{-1}(z / r)}^{\phi+\pi-\sin ^{-1}(z / r)}\left[\beta_{1} \beta_{2}(\theta, r, z) \ldots \beta_{n-1} \beta_{n}(\theta, r, z)\right] d \theta d r d z\left[\begin{array}{c}
\alpha_{1} \alpha_{2} \\
\vdots \\
\alpha_{n-1} \alpha_{n}
\end{array}\right] .
\end{aligned}
$$

Equation (50) involves a summation of products of deterministic coefficients multiplied by random variables and yields the energy release rate for a given probable fracture surface. Having samples of the random variables leads to the determination of the energy release rate PDF for any direction of propagation and length since the deterministic coefficients depend only on $\phi, \delta \alpha$, and $\underline{x}_{c}$.

Assuming now that the micromodulus function is of the form of (31), the coefficient $c$ of (15) can be taken as a random process such as

$$
c(r, \vartheta)=\tilde{c}_{0}+\hat{c}(r, \vartheta)
$$

where $\tilde{c}_{0}$ is the mean value.

The stiffness density of (33) and (34) involve integration over the horizon $\delta$. Thus, the random process can be decomposed by the Karhunen-Loève expansion in a way extensively described in stochastic finite element methods (SFEM) in [Ghanem and Spanos 1991]. In this study, the Monte Carlo simulation (MCS) technique is pursued for the determination of the energy PDF, and thus, the random process shown in (51) is sampled for the construction of the stiffness density. Due to the computational intensity of MCS, only the static case with initial fracture inside the domain is addressed in this study. In this context, (47), which involves the inversion of a random matrix, is obtained using the inverse Neumann expansion used in SFEM [Ghanem and Spanos 1991]. This yields

$$
\left[\begin{array}{c}
a_{11} \\
d_{11} \\
\vdots \\
a_{21} \\
d_{21} \\
\vdots
\end{array}\right]=\sum_{j=0}^{N_{e}}\left[\left[\begin{array}{ccc}
\sum_{i} \widetilde{K}_{G i} & \sum_{j} \widetilde{K}_{Q i} \\
\sum_{j} Q_{j}^{T} & 0
\end{array}\right]^{-1}\left[\begin{array}{ccc}
\sum_{i} K_{G i} & \sum_{j} K_{Q i} \\
\sum_{j} Q_{j}^{T} & 0
\end{array}\right]\right]^{j}\left[\begin{array}{cc}
\sum_{i} \widetilde{K}_{G i} & \sum_{j} \widetilde{K}_{Q i} \\
\sum_{j} Q_{j}^{T} & 0
\end{array}\right]^{-1}\left[\begin{array}{c}
b \\
0
\end{array}\right],
$$

where $N_{e}$ is the order of the Neumann expansion, $\widetilde{K}_{G}$ and $\widetilde{K}_{Q}$ are the average stiffness densities corresponding to the mean value of the random field, and $K_{G}$ and $K_{Q}$ are the stiffness densities corresponding to the zero mean random field. Note that a quite large sample of the coefficients must be calculated by pertinent MC simulations in order for the PDF of the energy release rate to be available for all lengths and angles of the crack propagation.

6.3. Monte Carlo application. For the example shown in Figure 9 with initial crack of $2 \mathrm{~mm}$ in the middle of the domain, $\delta \alpha=1 \mathrm{~mm}$, the PDF of the energy release rate at angles $\phi=\pi$ and $\phi=3 \pi / 4$ is 


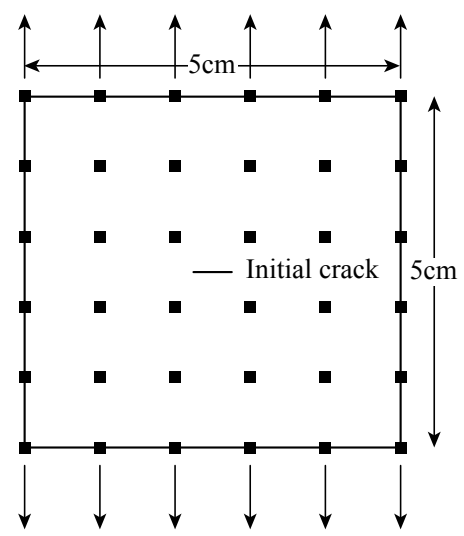

Figure 9. A $5 \mathrm{~cm} \times 5 \mathrm{~cm}$ plate under tension, including an initial crack in the center.

calculated by extensive MC simulations of (50) and (52). White Gaussian noise is used for the random process describing the micromodulus coefficient $c$ with mean value obtained from (16) for bulk modulus $k=1 \mathrm{~N} / \mathrm{cm}^{2}$. The horizon is discretized using 100 points, and thus 100 identical independent Gaussian random variables are considered. The inversion of the random matrix is obtained by a fourth-order Neumann expansion and the loading is perturbed by an additional white Gaussian noise with standard deviation $20 \%$ of the mean value which is taken to be equal to $1 \mathrm{~N} / \mathrm{cm}^{2}$.

After a large enough sample of the coefficients is obtained by pertinent MCS of (52), the probability density function of fracture in omnidirectional propagation orientations can be calculated by the deterministic integration of the coefficients of (50) for any angle $\phi$, propagation length $\delta \alpha$, and initial crack tip point $\underline{x}_{c}$. Figure 10 shows one realization of the random micromodulus coefficient with variance $20 \%$ of the mean value shown in (51). Figure 11 summarizes the results for the two approaches. The state of the art mesh-free numerical method is considered the method described in [Silling and Askari 2005].

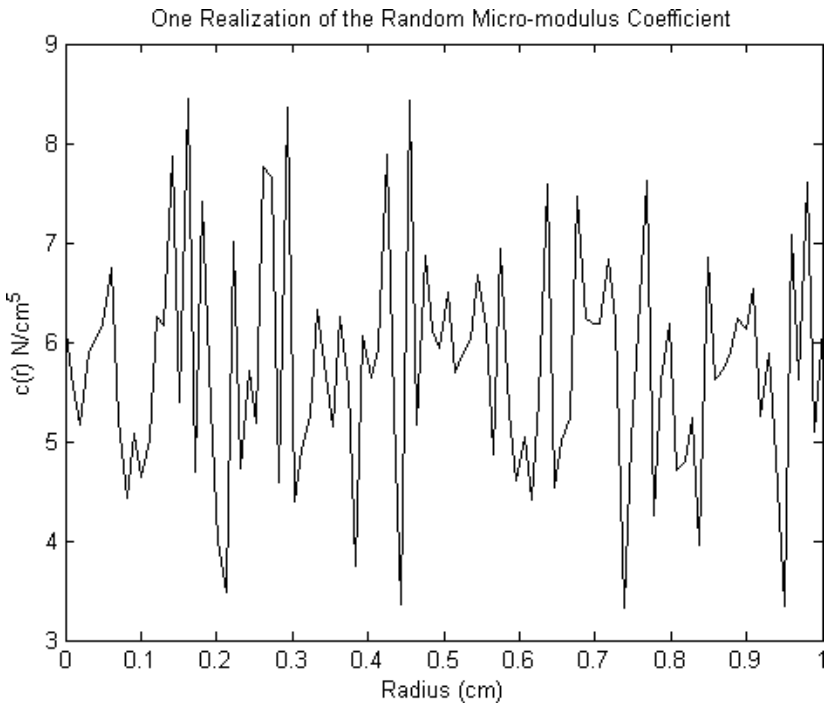

Figure 10. Random micromodulus coefficient spanning the horizon from 0 to $\delta$. 


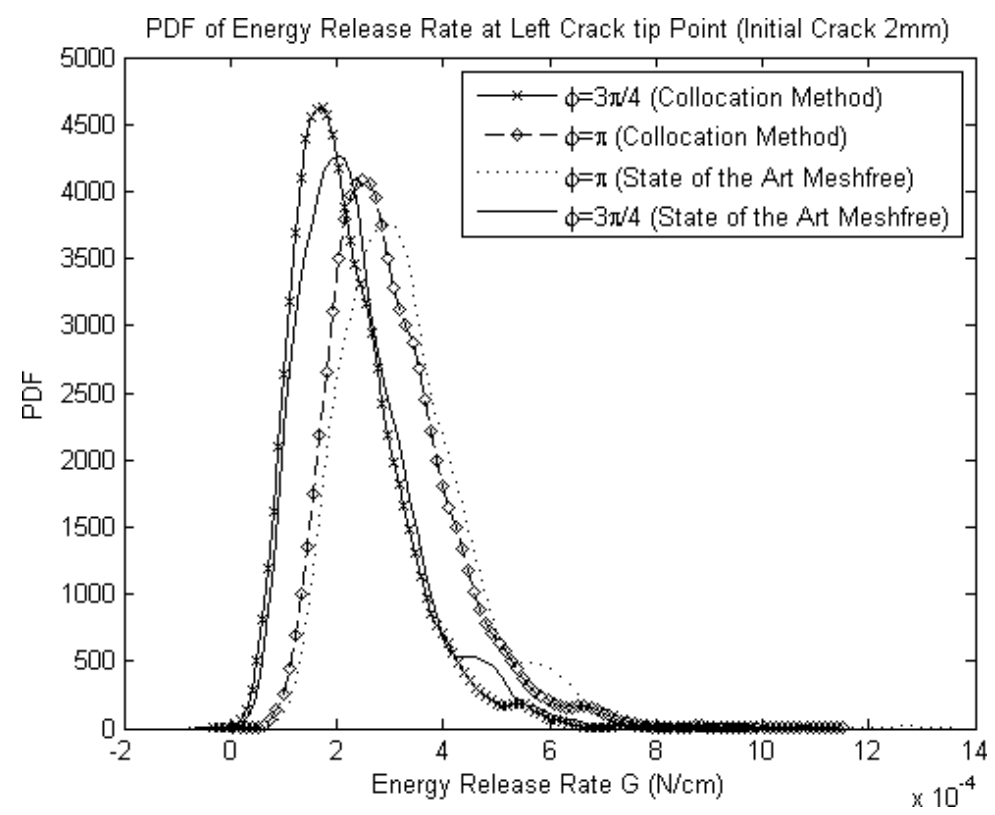

Figure 11. The PDF of the energy release rate for the left crack tip point estimated by 6000 Monte Carlo simulations.

The grid used for the mesh-free numerical method is $\Delta x=0.01 \mathrm{~cm}$ and thus 10201 nodes have been used. The horizon $\delta=5 \Delta x$ and the force constant $c$ is sampled from a Gaussian distribution.

The PDFs obtained from $6000 \mathrm{MC}$ simulations using the two methods are in reasonable agreement. However, the difference of the PDFs shown in Figure 11 is attributed to the fact that the methods do not produce identical results around the crack tip. Obviously, a finer grid of nodes will produce more accurate results for the Kansa collocation method. Further, for slightly different cracks inside the element the results deviate from the ones obtained by closed-form solutions. A possible resolution of this issue is the refinement of the grid by adding more nodes on the cracked element. However, in this paper, the introduction of a novel spatial discretization for deterministic/stochastic peridynamic modeling is the primary concern, and further work is warranted towards the direction of specifically describing the enrichment efficiency vis a vis the node refinement. In this model, node refinement was not considered due to the nature and simplicity of the numerical example and due to the fact that the enrichment of the $q$ basis [Fleming et al. 1997] provided quite accurate results for the specific example. However, for more elaborate cracks and medium shapes, node refinement is the most convenient option to achieve accuracy at the expense of additional computation cost.

\section{Concluding remarks}

In this work the application of peridynamic modeling to stochastic systems incorporating discontinuities has been considered. A novel approach for the spatial discretization of the integrodifferential equation arising from peridynamic theory that allows for stochastic extension has been devised. This spatial discretization has been based on the inverse multiquadric radial basis functions enriched with polynomials 
from the finite element method. Due to this formulation the stochastic problem has been solved in a manner similar to the stochastic finite element method, with some minor differences arising from the peridynamic stiffness density formulation. Following this similar path to the finite element method, the stiffness density of each element surrounded by four nodes has been constructed incorporating the fracture inside. The discontinuity inside the element has been handled by applying the visibility criterion and by changing the integration limits in the polar coordinate system. After formulating the stiffness density of the healthy and cracked elements, the global stiffness density matrix on the collocation points has been assembled and the system has become readily solvable. Next, the idea that the crack propagates in such a way that the energy release rate attains its maximum value has been adopted. In this context, since a continuous approximation has been devised for the displacements inside the domain, the energy release rate has been calculated progressively around the crack tip. For systems with random material properties under random excitations the PDF of the energy release rate has been obtained by Monte Carlo simulation of the requisite Neumann expansion. Further, having the PDF of the energy release for the area around the crack tip determines whether the crack propagates and in which direction with respect to the initial crack surface. Furthermore, this formulation has the advantage that the equilibrium equation is formed in terms of force density and displacement. Thus, there is no need for a double integration over the discontinuous medium. In the preceding regard, this is the first paper correlating the peridynamic formulation with reliability of failure. Specifically, stochastic fracture propagation and stochastic response of systems modeled utilizing the peridynamic formulation has been presented and results have demonstrated the effectiveness of the proposed approach.

\section{Appendix A: Numerical integration for stiffness determination}

For elements that do not include fracture or any kind of discontinuity the integrations of (33) are carried out by Gaussian quadrature using $2 \times 2$ points. Figure A.1 shows the Gaussian quadrature points.

Integrating the function $f(r, \theta)$ on a $2 \mathrm{D}$ rectangular domain $H=\left[\theta_{1}, \theta_{2}\right] \times\left[r_{1}, r_{2}\right]$ yields

$$
\int_{r_{1}}^{r_{2}} \int_{\theta_{1}}^{\theta_{2}} f(r, \theta) d r d \theta=\frac{r_{2}-r_{1}}{2} \frac{\theta_{2}-\theta_{1}}{2} \int_{-1}^{1} \int_{-1}^{1} f\left(\frac{r_{2}-r_{1}}{2} \hat{r}+\frac{r_{2}+r_{1}}{2}, \frac{\theta_{2}-\theta_{1}}{2} \hat{\theta}+\frac{\theta_{2}+\theta_{1}}{2}\right) d \hat{r} d \hat{\theta} .
$$

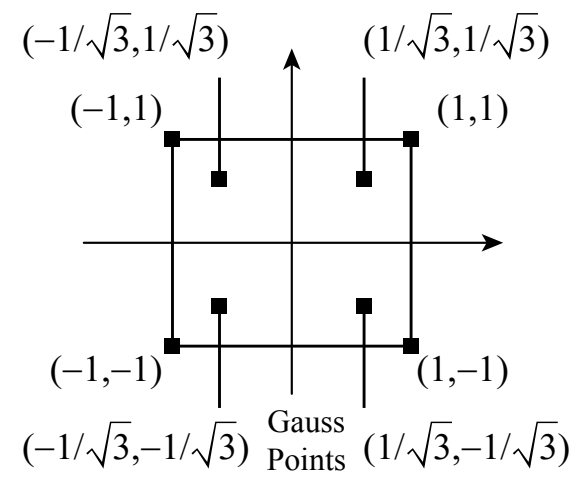

Figure A.1. Gaussian quadrature of $2 \times 2$ points of weight 1 . 
Applying Gaussian quadrature of $2 \times 2$ points for the numerical integration of (A.1) yields

$$
\int_{r_{1}}^{r_{2}} \int_{\theta_{1}}^{\theta_{2}} f(r, \theta) d r d \theta=\frac{r_{2}-r_{1}}{2} \frac{\theta_{2}-\theta_{1}}{2} \sum_{i, j=1}^{2}\left(\frac{r_{2}-r_{1}}{2} \hat{r}_{i}+\frac{r_{2}+r_{1}}{2}, \frac{\theta_{2}-\theta_{1}}{2} \hat{\theta}_{i}+\frac{\theta_{2}+\theta_{1}}{2}\right) .
$$

Next, for elements including fracture and discontinuity, numerical integration on a refined grid of points is needed. Figure A.2 shows an example of a refined grid.

The accurate determination of the fracture contour in polar coordinates is considered herein; specifically the determination of the curve $A B$ shown in Figure A.2. For the purposes of elucidation consider the initial crack shown in Figure A.3. Next, the position of the initial crack is known, therefore the vectors shown in Figure A.3 can be obtained; $\underline{z}_{1}$ and $\underline{z}_{2}$ are the vectors from the node of interest to the crack tips and $\underline{u}$ is the vector aligned with the crack with orientation from one crack tip to the other. The

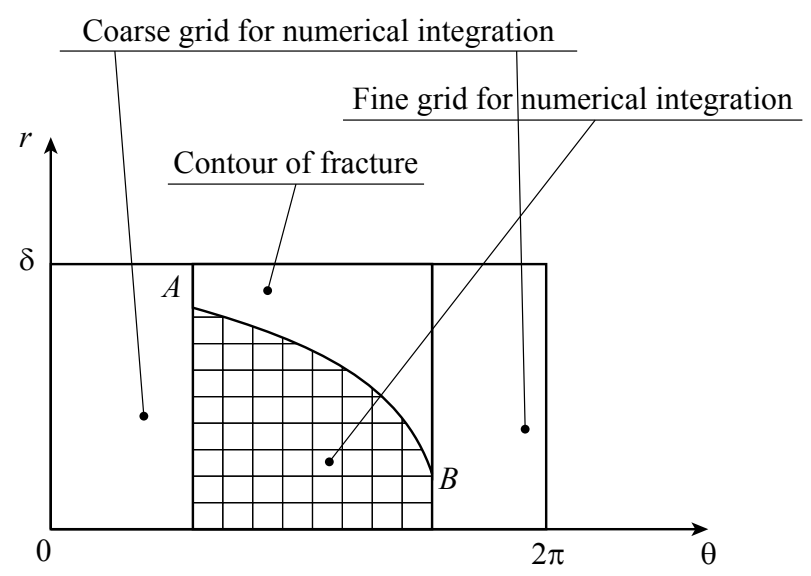

Figure A.2. Refined discretization for the numerical evaluation of the stiffness density of a fractured element.

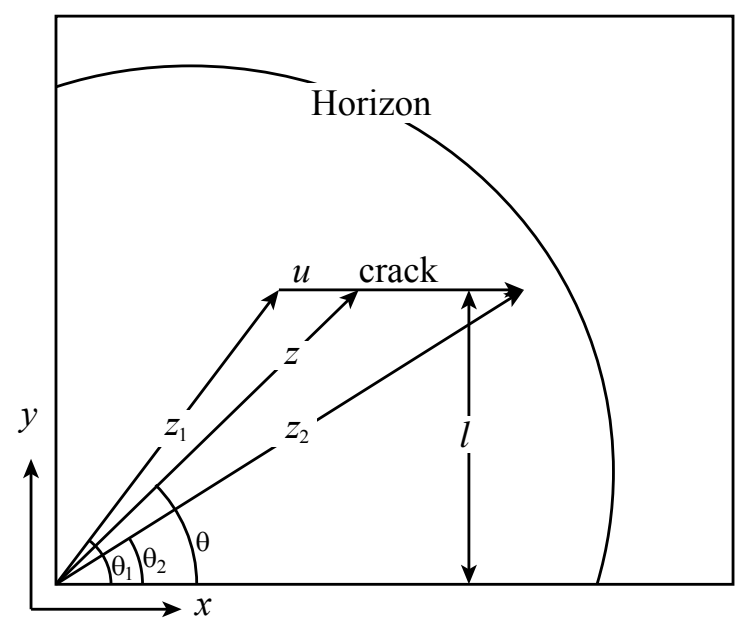

Figure A.3. Determination of the fractured contour of a cracked element. 
tangent of the angle of a vector $\underline{z}$ shown in Figure A.3 is given by

$$
\tan \theta(a)=\frac{l}{\mid \underline{z_{1}\left|\cos \theta_{1}+\alpha\right| \underline{u} \mid},}
$$

where $\alpha$ is a coefficient such that $\alpha \in[0,1]$. Next, the partial derivative of (A.3) with respect to the coefficient $\alpha$ yields

$$
\frac{d \theta}{d \alpha} \frac{1}{\cos ^{2} \theta(\alpha)}=-\frac{l|\underline{u}|}{\left(\left|\underline{z}_{1}\right| \cos \theta_{1}+\alpha|\underline{u}|\right)^{2}} .
$$

Carrying out the calculations yields a simplified expression for (A.4):

$$
d \theta=-\frac{l|\underline{u}|}{\left(\left|\underline{z}_{1}\right| \cos \theta_{1}+\alpha|\underline{u}|\right)^{2}+l^{2}} d \alpha .
$$

Obviously, as the vector $\alpha \vec{u}$ approaches the crack tip on the right, the rate of the angle is changing. Thus, the curve $A B$ shown in Figure A.2 is obtained numerically by fixing the $d \alpha$ value. Specifically, for a certain $d \alpha$ value, $\alpha_{j}$ is defined as

$$
\alpha_{j}=j \cdot d \alpha
$$

where $j=1, \ldots, 1 / d \alpha$ and thus $d \theta_{j}$ is defined as

$$
d \theta_{j}=-\frac{l|\underline{u}|}{\left(\left|\underline{z}_{1}\right| \cos \theta_{1}+\alpha_{j}|\underline{u}|\right)^{2}+l^{2}} d \alpha .
$$

Numerically integrating (A.7) by fixing $d \alpha$ yields

$$
\theta_{j}=\theta_{1}-\sum_{j} \frac{l|\underline{u}|}{\left(\left|\underline{z}_{1}\right| \cos \theta_{1}+\alpha_{j}|\underline{u}|\right)^{2}+l^{2}} d \alpha .
$$

Next, the corresponding length $r_{j}$ to the angle $\theta_{j}$ shown in Figure A.2 yields

$$
r_{j}=\left|\underline{z}_{1}+\alpha_{j} \underline{u}\right| .
$$

In this way the curve $A B$ is obtained as points $\theta_{j}$ and $r_{j}$ for $\alpha_{j} \in[0,1]$, and thus the contour of integration is determined for the numerical integration. For purposes of elucidation, points $A$ and $B$ in Figure A.2 correspond to the crack tips $\underline{z}_{2}$ and $\underline{z}_{1}$ shown in Figure A.3. In the same way (34) is numerically evaluated for elements including cracks. However, for healthy elements, polynomial functions $q$, and the micromodulus function shown in (31), the integration has a closed-form solution. Specifically, consider the first four linear functions of FEM as the $q$ functions

$$
q_{1}(\underline{x})=1, \quad q_{2}(\underline{x})=x, \quad q_{3}(\underline{x})=y, \quad q_{4}(\underline{x})=x y .
$$

Combining (29), (34), and (A.10) yields

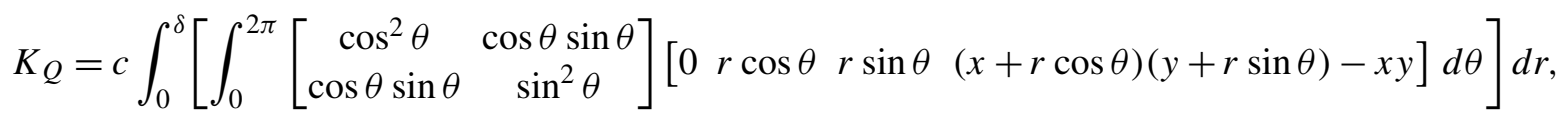

expanding which yields

$$
K_{Q}=c\left[\begin{array}{llll}
K_{Q 1} & K_{Q 2} & K_{Q 3} & K_{Q 4}
\end{array}\right]
$$


where

$$
\begin{gathered}
K_{Q 1}=\left[\begin{array}{ll}
0 & 0 \\
0 & 0
\end{array}\right], \quad K_{Q 2}=\left.\left.\left[\begin{array}{cc}
\frac{\cos ^{2} \theta \sin \theta}{3}+\frac{2 \sin \theta}{3} & -\frac{\cos ^{3} \theta}{3} \\
-\frac{\cos ^{3} \theta}{3} & \frac{\sin ^{3} \theta}{3}
\end{array}\right]\right|_{0} ^{2 \pi} \frac{r^{2}}{2}\right|_{0} ^{\delta}, \\
K_{Q 3}=\left.\left.\left[\begin{array}{cc}
-\frac{\cos ^{3} \theta}{3} & \frac{\sin ^{3} \theta}{3} \\
\frac{\sin ^{3} \theta}{3} & \frac{\cos \theta \sin ^{2} \theta}{3}-\frac{2 \cos \theta}{3}
\end{array}\right]\right|_{0} ^{2 \pi} \frac{r^{2}}{2}\right|_{0} ^{\delta}
\end{gathered}
$$

and finally

$$
K_{Q 4}=\left.\left.\left[\begin{array}{cc}
-\frac{\cos ^{4} \theta}{4} & -\frac{\sin ^{2} \theta \cos ^{3} \theta}{4}+\frac{\theta}{8}+\frac{\sin 2 \theta}{16} \\
-\frac{\sin ^{2} \theta \cos ^{3} \theta}{4}+\frac{\theta}{8}+\frac{\sin 2 \theta}{16} & \frac{\sin ^{4} \theta}{4}
\end{array}\right]\right|_{0} ^{2 \pi} \frac{r^{3}}{3}\right|_{0} ^{\delta}+x K_{Q 3}+y K_{Q 2} .
$$

\section{Appendix B: Kansa collocation method}

The Kansa collocation method in $1 \mathrm{D}$, for a set of $N=L / \Delta x+1$ equally spaced grid points of distance $\Delta x$, yields the equations in the following form:

$$
\left[\begin{array}{c}
u(0) \\
u(\Delta x) \\
u(2 \Delta x) \\
\vdots \\
0 \\
0
\end{array}\right]=\left[\begin{array}{cccccc}
g_{1}(0) & g_{1}(0) & \ldots & g_{N}(0) & q_{0}(0) & \ldots \\
g_{1}(\Delta x) & g_{1}(\Delta x) & \ldots & g_{N}(\Delta x) & q_{0}(\Delta x) & \ldots \\
g_{1}(2 \Delta x) & g_{1}(2 \Delta x) & \ldots & g_{N}(2 \Delta x) & q_{0}(2 \Delta x) & \ldots \\
\vdots & \vdots & \vdots & \vdots & \vdots & \vdots \\
q_{1}(0) & q_{1}(\Delta x) & q_{1}(2 \Delta x) & \ldots & 0 & 0 \\
\vdots & \vdots & \vdots & \vdots & 0 & 0
\end{array}\right]\left[\begin{array}{c}
a_{11} \\
a_{12} \\
\vdots \\
a_{1 N} \\
a_{21} \\
\vdots
\end{array}\right]
$$

The last $M$ rows represent the regularization equations of the coefficients which state that

$$
\sum_{k=1}^{N} q_{j}\left(\underline{x}_{k}\right) d_{2 k}=0 \quad \text { and } \quad \sum_{k=1}^{N} q_{j}\left(\underline{x}_{k}\right) d_{2 k}=0 \quad \text { for } \quad j=1, \ldots, M,
$$

where $M$ is the number of the additional basis functions $q$.

Assuming that the displacements of a point in the domain of Figure B.1 can be approximated by the adjacent nodes, (26) yields the displacement for a point inside the upper right square:

$$
\begin{aligned}
& u\left(\underline{x}_{i}+\underline{r}\right)=\sum_{k=i}^{i+3} a_{1 k} g_{k}\left(\underline{x}_{i}+\underline{r}\right)+\sum_{j=1}^{M} a_{2 j} q_{j}\left(\underline{x}_{i}+\underline{r}\right), \\
& v\left(\underline{x}_{i}+\underline{r}\right)=\sum_{k=i}^{i+3} d_{1 k} g_{k}\left(\underline{x}_{i}+\underline{r}\right)+\sum_{j=1}^{M} d_{2 j} q_{j}\left(\underline{x}_{i}+\underline{r}\right) .
\end{aligned}
$$




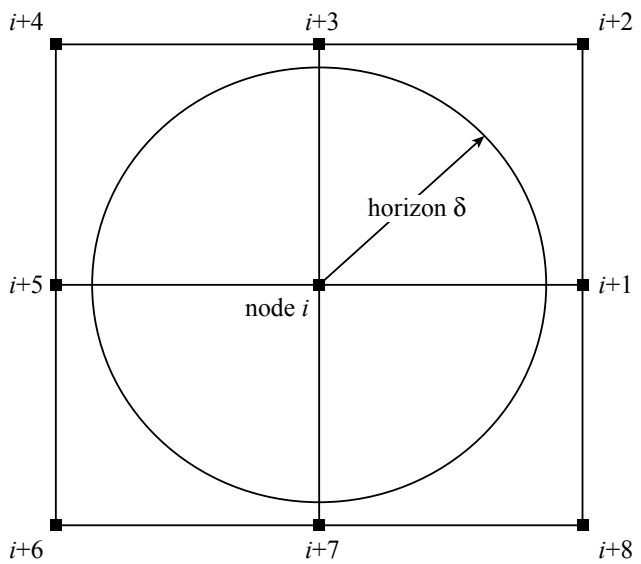

Figure B.1. The horizon of one node over four elements, the stiffness density of the node $i$ is calculated through (33) and (34).
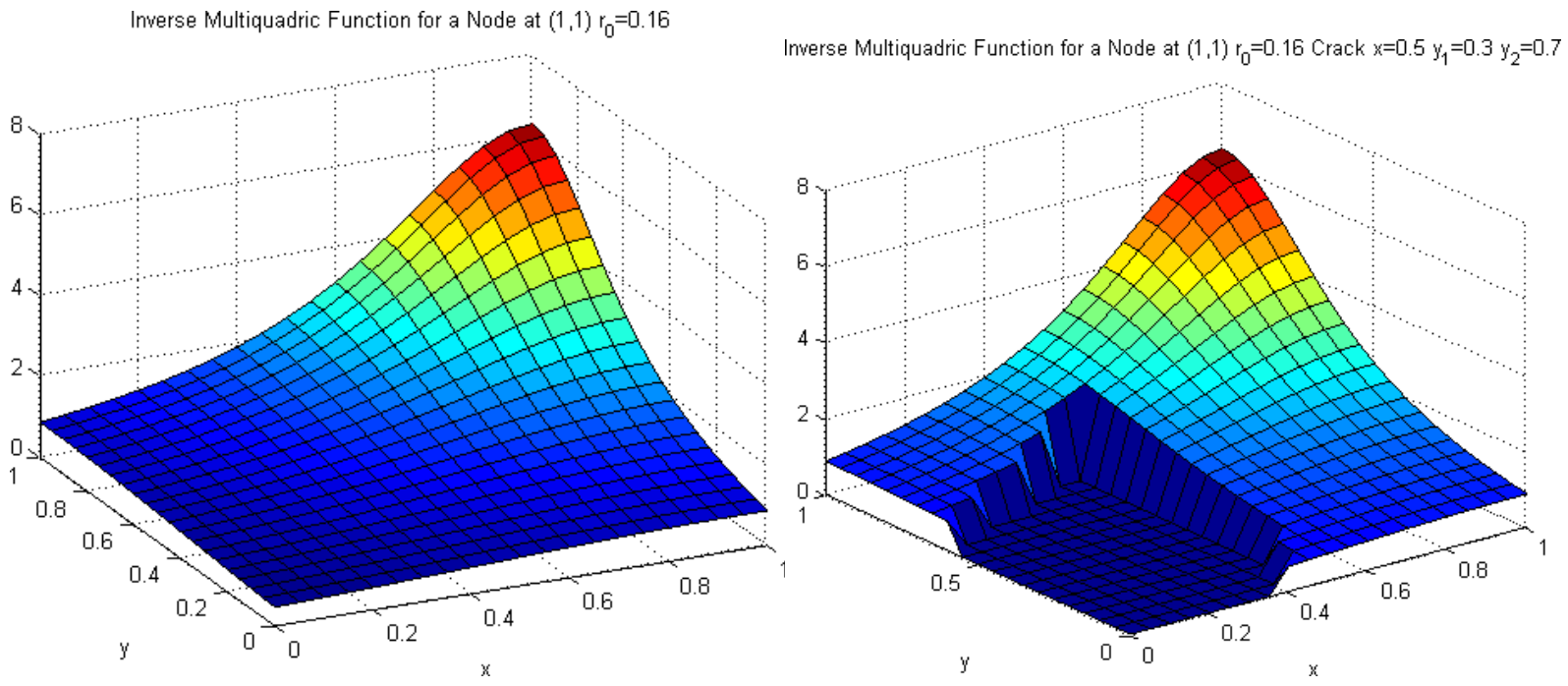

Figure B.2. IMQ basis function of the corresponding node on a four-node element, with and without a crack inside the horizon.

Figure B.2 shows the IMQ function for the same node, both for a healthy and a cracked four-node element. It can be seen that the visibility criterion is applied on the cracked element since the crack disrupts the horizon of the node.

\section{Appendix C: Linearized pairwise force function}

The linearized pairwise force function as introduced in [Silling 2000] imposes an implicit assumption of small rigid body rotations. Specifically, consider Equation (12), which defines the stretch of a bond. 


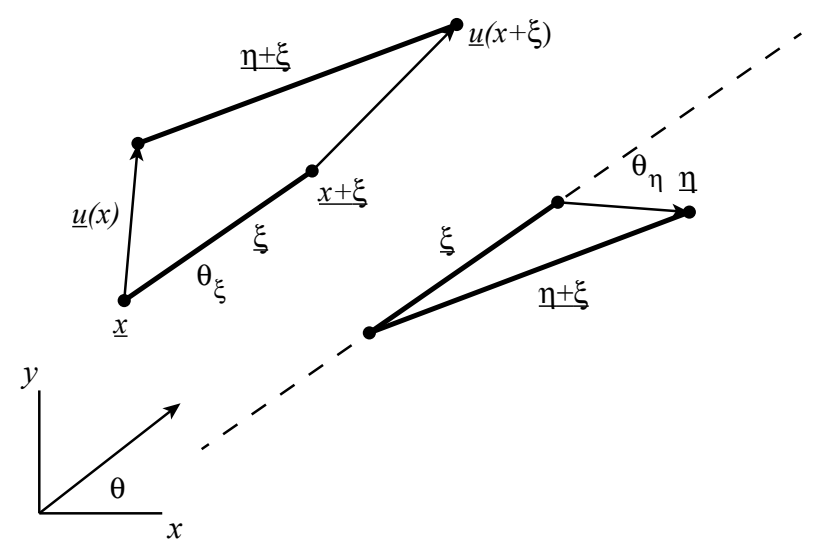

Figure C.1. One bond before and after deformation.

Carrying out the calculations yields

$$
s=\left|1+\frac{\eta}{\underline{\xi}}\right|-1
$$

Next, using complex notation for the vectors yields

$$
\underline{\eta}=|\underline{\eta}|\left(\cos \theta_{\eta}+i \sin \theta_{\eta}\right), \quad \underline{\xi}=|\underline{\xi}|\left(\cos \theta_{\xi}+i \sin \theta_{\xi}\right),
$$

where $\theta_{\eta}$ and $\theta_{\xi}$ are the angles of the vectors with respect to the orthogonal reference system. Figure C.1 helps elucidate the concept. Next, combining both parts of (C.2) yields

$$
\frac{\underline{\eta}}{\underline{\xi}}=\frac{|\underline{ }|}{|\underline{\xi}|}\left(\cos \left(\theta_{\eta}-\theta_{\xi}\right)+i \sin \left(\theta_{\eta}-\theta_{\xi}\right)\right) \text {. }
$$

Obviously the angles of the vectors determine if the vector division yields a vector or a scalar. For the case of

$$
\theta_{\eta} \simeq \theta_{\xi}
$$

(C.3) yields

$$
\frac{\underline{\eta}}{\underline{\xi}} \simeq \frac{|\underline{\eta}|}{|\underline{\xi}|}
$$

and (C.1) becomes

$$
s \simeq \frac{|\underline{\eta}|}{|\underline{\xi}|}
$$

Equation (C.6) implicitly assumes that the deformation of the bond is collinear with the bond's initial orientation or that the bond exhibits small rotation which can be neglected.

\section{References}

[Belytschko et al. 1994] T. Belytschko, L. Gu, and Y. Y. Lu, "Fracture and crack growth by element free Galerkin methods", Model. Simul. Mater. Sci. Eng. 2:3A (1994), 519-534.

[Belytschko et al. 1995] T. Belytschko, Y. Y. Lu, and L. Gu, "Crack propagation by element-free Galerkin methods", Eng. Fract. Mech. 51:2 (1995), 295-315. 
[Bobaru 2007] F. Bobaru, "Influence of van der Waals forces on increasing the strength and toughness in dynamic fracture of nanofibre networks: a peridynamic approach”, Model. Simul. Mater. Sci. Eng. 15:5 (2007), 397-417.

[Bobaru et al. 2009] F. Bobaru, M. Yang, L. F. Alves, S. A. Silling, E. Askari, and J. Xu, "Convergence, adaptive refinement, and scaling in 1D peridynamics", Int. J. Numer. Methods Eng. 77:6 (2009), 852-877.

[Emmrich and Weckner 2007a] E. Emmrich and O. Weckner, "On the well-posedness of the linear peridynamic model and its convergence towards the Navier equation of linear elasticity", Commun. Math. Sci. 5:4 (2007), 851-864.

[Emmrich and Weckner 2007b] E. Emmrich and O. Weckner, "The peridynamic equation and its spatial discretisation", Math. Model. Anal. 12:1 (2007), 17-27.

[Eringen et al. 1977] A. C. Eringen, C. G. Speziale, and B. S. Kim, "Crack-tip problem in non-local elasticity”, J. Mech. Phys. Solids 25:5 (1977), 339-355.

[Fleming et al. 1997] M. Fleming, Y. A. Chu, B. Moran, and T. Belytschko, "Enriched element-free Galerkin methods for crack tip fields", Int. J. Numer. Methods Eng. 40:8 (1997), 1483-1504.

[Gdoutos 1990] E. E. Gdoutos, Fracture mechanics: criteria and applications, edited by G. C. Sih, Engineering Application of Fracture Mechanics 10, Kluwer Academic, Dordrecht, 1990.

[Ghanem and Spanos 1991] R. G. Ghanem and P. D. Spanos, Stochastic finite elements: a spectral approach, Springer, New York, 1991.

[Kansa 1990a] E. J. Kansa, "Multiquadrics—a scattered data approximation scheme with applications to computational fluiddynamics, I: Surface approximations and partial derivative estimates", Comput. Math. Appl. 19:8-9 (1990), 127-145.

[Kansa 1990b] E. J. Kansa, "Multiquadrics—a scattered data approximation scheme with applications to computational fluiddynamics, II: Solutions to parabolic, hyperbolic and elliptic partial differential equations", Comput. Math. Appl. 19:8-9 (1990), $147-161$.

[Kröner 1967] E. Kröner, "Elasticity theory of materials with long range cohesive forces", Int. J. Solids Struct. 3:5 (1967), 731-742.

[Macek and Silling 2007] R. W. Macek and S. A. Silling, "Peridynamics via finite element analysis", Finite Elem. Anal. Des. 43:15 (2007), 1169-1178.

[Silling 2000] S. A. Silling, "Reformulation of elasticity theory for discontinuities and long-range forces", J. Mech. Phys. Solids 48:1 (2000), 175-209.

[Silling and Askari 2005] S. A. Silling and E. Askari, "A meshfree method based on the peridynamic model of solid mechanics", Comput. Struct. 83:17-18 (2005), 1526-1535.

[Silling et al. 2003] S. A. Silling, M. Zimmermann, and R. Abeyaratne, "Deformation of a peridynamic bar", J. Elasticity 73:1-3 (2003), 173-190.

[Silling et al. 2007] S. A. Silling, M. Epton, O. Weckner, J. Xu, and E. Askari, "Peridynamic states and constitutive modeling", J. Elasticity 88:2 (2007), 151-184.

[Warren et al. 2009] T. L. Warren, S. A. Silling, A. Askari, O. Weckner, M. A. Epton, and J. Xu, "A non-ordinary state-based peridynamic method to model solid material deformation and fracture", Int. J. Solids Struct. 46:5 (2009), 1186-1195.

[Zhou and Du 2010] K. Zhou and Q. Du, "Mathematical and numerical analysis of linear peridynamic models with nonlocal boundary conditions", SIAM J. Numer. Anal. 48:5 (2010), 1759-1780.

[Zi and Belytschko 2003] G. Zi and T. Belytschko, "New crack-tip elements for XFEM and applications to cohesive cracks", Int. J. Numer. Methods Eng. 57:15 (2003), 2221-2240.

Received 27 Aug 2010. Revised 28 Feb 2011. Accepted 10 Apr 2011.

Georgios I. Evangelatos: Georgios. Evangelatos@bp.com

Department of Civil and Environmental Engineering, Rice University, 6100 Main Street, Mail Stop 318, Houston, TX 77005-1892, United States

Pol D. SPANos: spanos@rice.edu

Departments of Civil and Mechanical Engineering, Rice University, 6100 Main Street, Mail Stop 321,

Houston, TX 77005-1892, United States 


\title{
JOURNAL OF MECHANICS OF MATERIALS AND STRUCTURES
}

\author{
jomms.org
}

Founded by Charles R. Steele and Marie-Louise Steele

EDITORS

Charles R. SteEle

DAVIDE BIGONI

Stanford University, USA

YASUHIDE SHINDO

University of Illinois at Urbana-Champaign, USA

Tohoku University, Japan

\section{EDITORIAL BOARD}

$\begin{aligned} \text { H. D. BUI } & \text { École Polytechnique, France } \\ \text { J. P. CARTER } & \text { University of Sydney, Australia } \\ \text { R. M. CHRISTENSEN } & \text { Stanford University, USA } \\ \text { G. M. L. GLADWELL } & \text { University of Waterloo, Canada } \\ \text { D. H. HODGES } & \text { Georgia Institute of Technology, USA } \\ \text { J. HUTCHINSON } & \text { Harvard University, USA } \\ \text { C. HWU } & \text { National Cheng Kung University, Taiwan } \\ \text { B. L. KARIHALOO } & \text { University of Wales, UK } \\ \text { Y. Y. KIM } & \text { Seoul National University, Republic of Korea } \\ \text { Z. MROZ } & \text { Academy of Science, Poland } \\ \text { D. PAMPLONA } & \text { Universidade Católica do Rio de Janeiro, Brazil } \\ \text { M. B. RUBIN } & \text { Technion, Haifa, Israel } \\ \text { A. N. SHUPIKOV } & \text { Ukrainian Academy of Sciences, Ukraine } \\ \text { T. TARNAI } & \text { University Budapest, Hungary } \\ \text { F. Y. M. WAN } & \text { University of California, Irvine, USA } \\ \text { P. WRIGGERS } & \text { Universität Hannover, Germany } \\ \text { W. YANG } & \text { Tsinghua University, China } \\ \text { F. ZIEGLER } & \text { Technische Universität Wien, Austria } \\ & \\ \text { PRODUCTION } & \text { contact@ msp.org } \\ \text { SILVIO LEVY } & \text { Scientific Editor }\end{aligned}$

Cover design: Alex Scorpan

Cover photo: Mando Gomez, www.mandolux.com

See http://jomms.org for submission guidelines.

JoMMS (ISSN 1559-3959) is published in 10 issues a year. The subscription price for 2011 is US \$520/year for the electronic version, and \$690/year (+\$60 shipping outside the US) for print and electronic. Subscriptions, requests for back issues, and changes of address should be sent to Mathematical Sciences Publishers, Department of Mathematics, University of California, Berkeley, CA 94720-3840.

JoMMS peer-review and production is managed by EditFLow ${ }^{\circledR}$ from Mathematical Sciences Publishers.

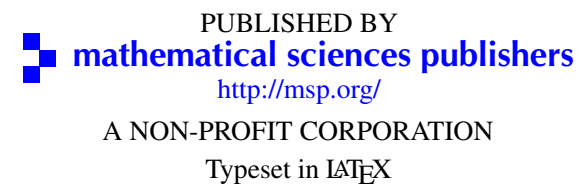

Copyright (C2011 by Mathematical Sciences Publishers 


\section{Journal of Mechanics of Materials and Structures}

\section{Volume 6, No. 7-8}

September-October 2011

\section{Special issue \\ Eleventh Pan-American Congress \\ of Applied Mechanics (PACAM XI)}

Preface

Adair R. Aguiar

949

Influence of specimen geometry on the Portevin-Le Châtelier effect due to dynamic strain aging

for the AA5083-H116 aluminum alloy

Rodrigo Nogueira de Codes and Ahmed Benallal

Dispersion relations for SH waves on a magnetoelectroelastic heterostructure with imperfect

interfaces

J. A. Otero, H. Calas, R. Rodríguez, J. Bravo, A. R. Aguiar and G. Monsivais

Numerical linear stability analysis of a thermocapillary-driven liquid bridge with magnetic stabilization

Yue Huang and Brent C. Houchens

Numerical investigation of director orientation and flow of nematic liquid crystals in a planar 1:4 expansion Pedro a. Cruz, Murilo F. Tomé, IAin W. Stewart and Sean McKee

Critical threshold and underlying dynamical phenomena in pedestrian-induced lateral vibrations of footbridges

Stefano LenCI and LAURA MARCHEgGiani

Free vibration of a simulation CANDU nuclear fuel bundle structure inside a tube

XUAN ZHANG and SHUdONG Yu

Nonlinear dynamics and sensitivity to imperfections in Augusti's model

D. Orlando, P. B. Gonçalves, G. Rega and S. LenCi

Active control of vortex-induced vibrations in offshore catenary risers: A nonlinear normal mode approach

CArlos E. N. MAZzilli and César T. SANCheS

Nonlinear electromechanical fields and localized polarization switching of piezoelectric macrofiber composites

Yasuhide Shindo, Fumio Narita, KoJi SATo and Tomo TAKeda

1089

Three-dimensional BEM analysis to assess delamination cracks between two transversely isotropic materials

Nicolás O. Larrosa, Jhonny E. Ortiz and Adrián P. Cisillino

Porcine dermis in uniaxial cyclic loading: Sample preparation, experimental results and modeling

A. E. Ehret, M. Hollenstein, E. MAzzA and M. Itskov

Analysis of nonstationary random processes using smooth decomposition

Rubens SAMpaio and Sergio Bellizzi

Perturbation stochastic finite element-based homogenization of polycrystalline materials

S. LePage, F. V. Stump, I. H. Kim and P. H. Geubelle

A collocation approach for spatial discretization of stochastic peridynamic modeling of fracture

Georgios I. Evangelatos and POL D. SPANOS 